\title{
Modeling Frequency Dependent Core Loss of Ferrite Materials using Permeance-Capacitance Analogy for System-Level Circuit Simulations
}

\author{
M. Luo, D. Dujic, and J. Allmeling
}

This material is posted here with permission of the IEEE. Such permission of the IEEE does not in any way imply IEEE endorsement of any of EPFL's products or services. Internal or personal use of this material is permitted. However, permission to reprint / republish this material for advertising or promotional purposes or for creating new collective works for resale or redistribution must be obtained from the IEEE by writing to pubs-permissions@ieee. org. By choosing to view this document, you agree to all provisions of the copyright laws protecting it. 


\title{
Modeling Frequency Dependent Core Loss of Ferrite Materials using Permeance-Capacitance Analogy for System-Level Circuit Simulations
}

\author{
Min Luo, Member, IEEE, Drazen Dujic, Senior Member, IEEE, and Jost Allmeling, Member, IEEE
}

\begin{abstract}
Ferrite core materials are preferred for magnetic components in power electronic converters considering their low cost and high permeability. Thanks to the negligible electrical conductivity, core loss is dominated by the frequency independent hysteresis effect in most operating conditions. In the applications of high switching frequency under certain modulation scheme, however, the core loss of ferrite materials do exhibit obvious frequency dependency, known as relaxation effect, which is strongly influenced by the magnetic flux's rate of change and can not be ascribed to material conductivity. In order to incorporate the core loss including both frequency dependent- and independent effect into system level simulation environment of power converters, so that the system efficiency can be pre-evaluated during the design-phase as reference for optimization, dynamic model which is physically intuitive and easy to parametrise is desired. This work proposes an approach to model the core loss of ferrite materials combining both frequency dependent relaxation effect and frequency independent hysteresis, which can be flexibly applied for various operation conditions with good accuracy.
\end{abstract}

Index Terms-core loss, frequency dependent, ferrite material, magnetic circuit, permeance-capacitance

\section{NOMENCLATURE}

$\begin{array}{ll}B & \text { Flux density. } \\ H & \text { Field strength. } \\ F & \text { Magnetomotive force. } \\ \Phi & \text { Magnetic flux. } \\ \mathscr{P} & \text { Magnetic permeance. } \\ \mu & \text { Magnetic permeability. }\end{array}$

\section{INTRODUCTION}

Ferrite is a popular type of core material to produce magnetic components in power electronic applications, taking advantage of its low cost and relatively high permeability. In order to predict the overall efficiency and determine the cooling condition, core loss of the ferrite based magnetic components need to be estimated during the design phase of the power converters. In general, the core loss of magnetic materials can be separated into three parts, according to [1]:

- Hysteresis loss: Frequency independent effect related to the material nonlinearity.

M. Luo and D. Dujic are with the Power Electronics Laboratory, École Polytechnique Fédérale de Lausanne (EPFL), Lausanne, CH-1015 Switzerland (e-mail:min.luo@epfl.ch,drazen.dujic@epfl.ch).

J. Allmeling is with Plexim GmbH, Zürich, CH-8005 Switzerland (e-mail: allmeling@plexim.com).

Manuscript received March 25, 2018; accepted June 29, 2018.
- Eddy current loss: Frequency dependent effect related to the material conductivity.

- Relaxation loss: Frequency dependent effect related to other physical mechanisms.

Due to the low electrical conductivity, eddy current which is obviously taking place in metal-based core materials (e.g. silicon steel) is negligible in ferrites, so that the core loss is dominated by frequency independent hysteresis effect in most operating conditions of power converters. However in high frequency (e.g. $>20 k H z$ ) applications under certain modulation schemes, the core loss of ferrite materials do exhibits strong frequency- and rate dependency, which can not be ascribed to eddy current but the relaxation effect.

In practice, the core loss is usually estimated using empirical formulas based on Steinmetz equation (SE), whose parameters are extracted from experimental measurements under sinusoidal excitation. Authors of [2] proposed an improved form of Steinmetz equation (iGSE) to generalize it to non-sinusoidal excitations, as is the case in power electronic applications. In the work of [3], the authors have observed that under phaseshift modulation (e.g. in the case of galvanically isolated type of DC-DC converters shown in Fig. 1) where a zero-voltage period is present, and strongly asymmetrical PWM with dutycycle far away from $50 \%$, the core loss increases significantly in comparison to the case of $50 \%$ symmetrical PWM. In the aforementioned modulation schemes, authors of [4] have demonstrated that the improved form proposed by [2] still significantly underestimated the core loss. Aiming to reduce the error, an additional term has been added to the iGSE, resulting in improved-improved generalized Steinmetz equation (iiGSE). Alternatively, the approach based on the composite waveform hypothesis $(\mathrm{CWH})$ proposed by [3] was further developed by [5]. Physically, this considerable relaxation loss under high switching frequency PWM excitation with zerovoltage period or strongly asymmetrical duty cycle, takes place if the thermal equilibrium of a magnetic system is suddenly altered by some external force and the reestablishment of a new thermal equilibrium happens progressively, as introduced in the work of [1]. More detailed analysis of relaxation effect can be found in [6].

Although the continuously improved empirical equations proposed by the existing publications provide even better accuracy in core loss estimation, the physics in behind are still obscure and unintuitive for power electronic engineers whose knowledge, unfortunately, is often not very deep in the area of magnetics. In the design phase of power converters, to help 

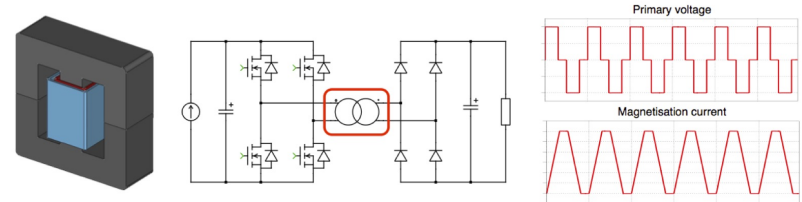

Fig. 1: Isolation transformer in DCDC converter with phaseshift modulation and the typical operational waveforms of primary voltage and magnetisation current.

engineers with understanding the physical interaction between the components and evaluating the dynamic behavior of the whole system, time-domain circuit simulation is commonly adopted. The empirical equations, however, are difficult to be incorporated into the circuit simulation environment. In order to reflect the core loss's impact on the dynamic behavior of the converter system, authors of [7] managed to interpret iGSE directly into SPICE-type circuit simulator via a variable resistor, which achieved good accuracy in various operating conditions. However the loss mechanism still remains as a black box having the same transparency level as the empirical equation, where all the three loss components (hysteresis, eddy current, relaxation) are mixed together.

To provide a clear insight of the loss mechanism, it's desired to present the loss components separately in the simulation environment. Frequency independent hysteresis loss can be exclusively represented in time domain by classical Preisach model in a flexible way [8]. In the work of [9] and [10], the classical Preisach model is extended in circuit simulation for static hysteresis effect of ferrite materials, where good accuracy was achieved in various excitation amplitudes. Beside using Preisach model for frequency independent hysteresis, the other two loss components - eddy current and residual effect are incorporated by the authors of [11] via introducing two resistive components. However the model was only verified under symmetrical PWM excitation without zerovoltage period, where the relaxation loss hardly has visible impact while the eddy current loss is intrinsically negligible in ferrite materials. Authors of [12] modeled the relaxation effect explicitly using a third-order circuit model, the similar model structure was also applied later by [13], while the frequency independent hysteresis effect was not taken into account and the fidelity is verified in frequency response rather than time domain.

Aiming to provide a model which is sufficiently accurate to capture the total core loss of ferrite materials, and is meanwhile clear and intuitive enough in terms of the individual loss mechanism, the modeling approach proposed by this work differs from the existing publications in the following aspects:

- Frequency dependent relaxation effect is specifically modeled. An explicit way of parametrization from measurement result is introduced.

- Frequency independent hysteresis effect is also individually covered using Preisach model, and combined in the same circuit structure.

- The total core loss is physically present in the circuit model, while the mechanism of individual loss compo- nents (hysteresis, relaxation) can be clearly investigated.

- The permeance-capacitor based magnetic circuit proposed by [14] and further adopted in the work of [15], [10] is chosen as the fundamental platform, which has seamless interface to electrical circuit. In permeance magnetic circuit, the relaxation effect can be explained in a intuitive way, as will be presented in the following sections.

In section II, the experimental result of operating condition, where both frequency independent hysteresis and frequency dependent relaxation effect are obviously visible, is presented. In section III, referring to the experimental result, the fundamental model structure and physic origins of the frequency dependent relaxation effect is interpreted into permeancemagnetic circuit, as starting point of the modeling. The parametrization process of the proposed model for ferrite material and the required measurement data are introduced in section IV. Afterwards in section V, the proposed model is verified by experimental test of different ferrite materials under various operating conditions including switching frequency, duty cycle and zero-voltage period. At the end, summary and outlook are provided in section VI.

\section{ExPERIMENTAl MEASUREMENT}

\section{A. Test setup}

For material characterization and model verification purpose, a multi-functional magnetic characterization test setup has been constructed, which is able to provide both low frequency sinusoidal and high frequency PWM excitations. The hardware of the characterization setup is designed to have $24 \mathrm{~V}$ rated output voltage and $6 \mathrm{~A}$ rated output current. The basic idea is derived from the V-I approach proposed by the work of [16]. The circuit schematic and photo of a actual setup are shown in Fig. 2 and Fig. $3 \mathrm{~b}$ respectively, and include the following elements:

- Controlled voltage source $V_{1}$ : Realized using power amplifier of type LM3886 with $2 \mathrm{MHz}$ gain-bandwidthproduct and is supplied by $\pm 24 \mathrm{~V}$. The main usage is to generate sinusoidal excitation.

- Single phase T-type bridge: Composed of three MOSFET switches (pairs) $\left(Q_{01} \& Q_{02}, Q_{1}, Q_{2}\right)$ to generate threelevel PWM excitation. Both $Q_{1}$ and $Q_{2}$ are connected to controlled voltage sources $\left(V_{3}, V_{4}\right)$ which are realized using the same type of power amplifier as $V_{1}$.

- Core sample: Following the V-I approach for magnetic material characterization, the core sample is equipped with two windings as demonstrated in Fig. 2. The primary winding is excited by the power stage whose current is measured to obtain field strength $H$. The secondary winding is left open, the voltage of which is measured and integrated in time domain to obtain flux density $B$.

- Current measurement: Realized using low-inductance shunt resistor. The voltage drop of the shunt resistor is sensed and amplified using differential instrumentation amplifiers. Two sets of shunt resistors (different resistance) circuitry are mounted to achieve different resolutions of measurement. 


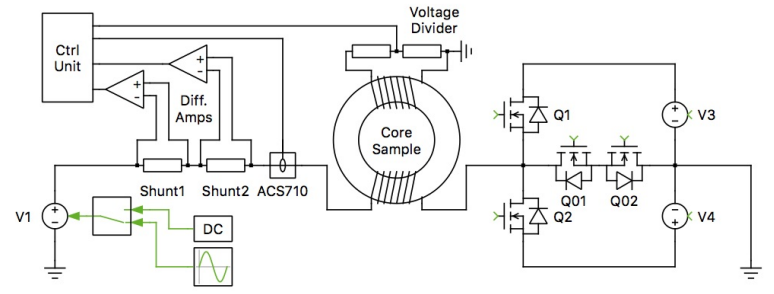

Fig. 2: Circuit schematic of the test setup.
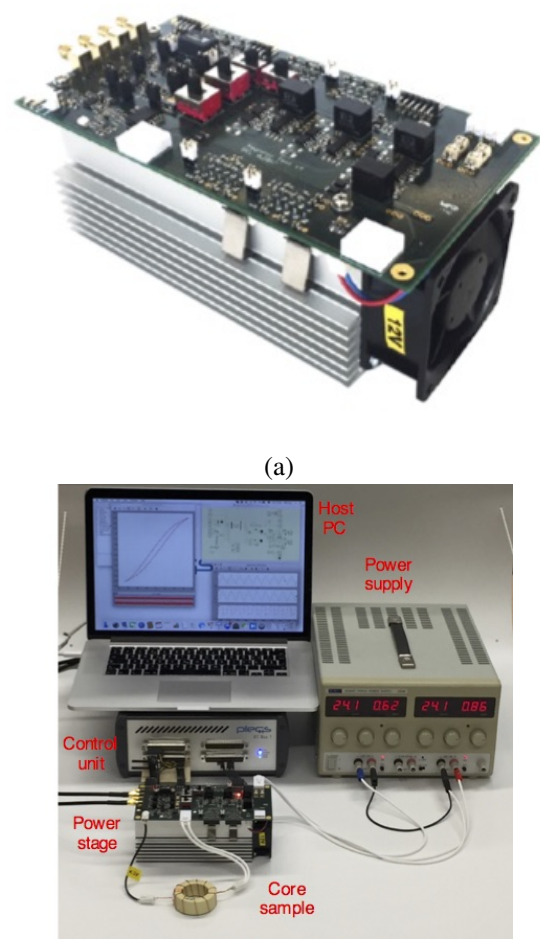

(b)

Fig. 3: (a) Power stage hardware of the test setup; (b) Complete configuration of the test setup including the power stage, core sample, power supply, control unit (PLECS RT-Box) and host PC.

- Voltage measurement: Realized using low capacitance resistive voltage divider. The scaled voltage is buffered and amplified by operational amplifier with $180 \mathrm{MHz}$ bandwidth.

- Control unit: One PLECS RT-Box is deployed as control unit for the test setup, which is graphically operated by a host PC.

\section{B. Measurement results}

To demonstrate the impact of relaxation effect at high switching frequency excitation, comparative measurement has been carried out based on the ferrite material N87 from EPCOS, with the toroidal core of size code "R $20.0 \times 10.0 \times$ 7.00 " chosen as sample. The turns number of both windings are $N_{1}=N_{2}=5$. As the temperature dependency is not in the scope of this work, all tests for parametrization and

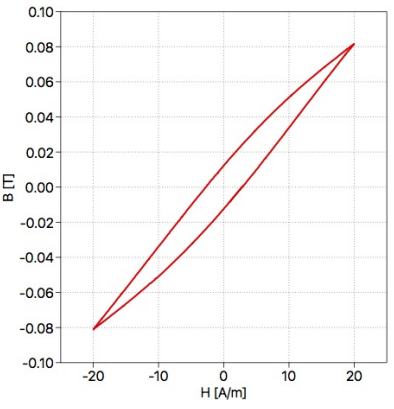

(a)

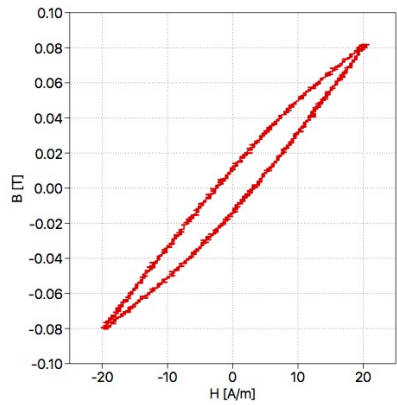

(b)
Fig. 4: Measured $B-H$ characteristic of ferrite N87 with peak strength $\hat{H}=20 \mathrm{~A} / \mathrm{m}$ excited by: (a) $500 \mathrm{~Hz}$ sinusoidal excitation; (b) $50 \mathrm{kHz}$ symmetrical PWM excitation without zero voltage period.

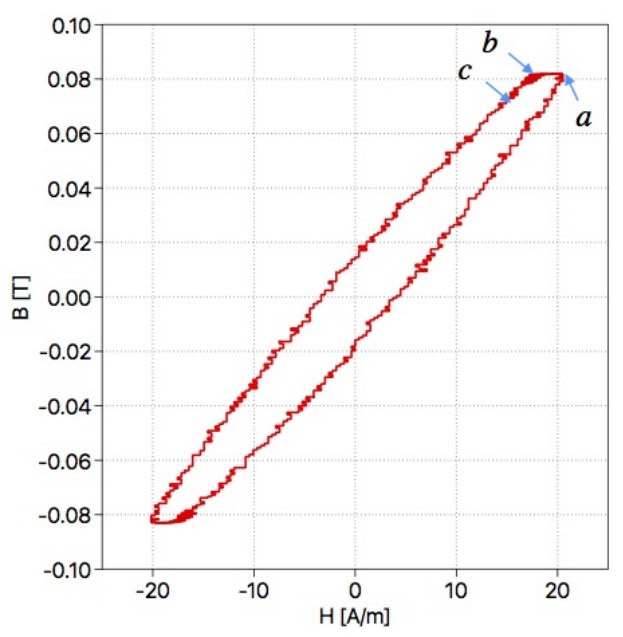

(a)

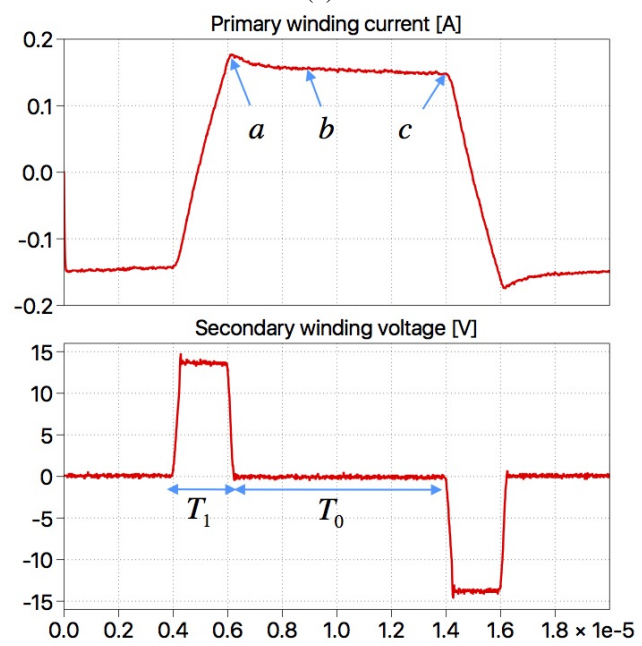

(b)

Fig. 5: Measurement results of ferrite N87 (peak strength $\hat{H}=$ $20 \mathrm{~A} / \mathrm{m}$ ) excited by $50 \mathrm{kHz}$ symmetrical PWM excitation with zero voltage period of $T_{0}=8 \mu s$ (a) $B-H$ characteristic (b) Primary winding current and secondary winding voltage 


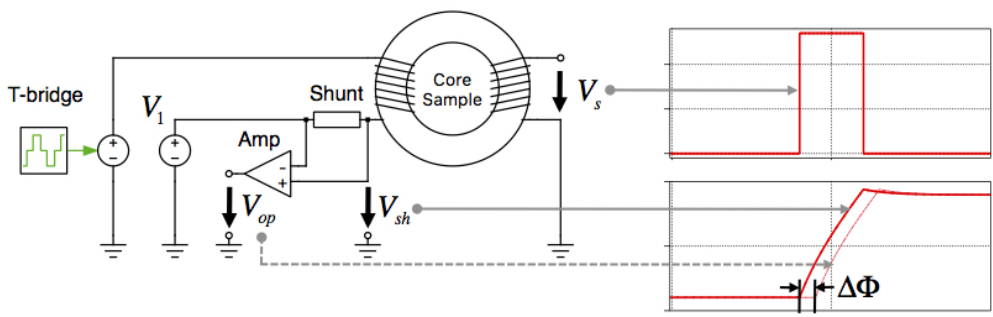

Fig. 6: Phase error compensation of the differential amplifier output in the current measurement.

verification have been conducted under room temperature of $25^{\circ} \mathrm{C}$.

At first, the core sample is excited by sinusoidal excitation generated from the controlled voltage source $V_{1}$ (with switches $Q_{01} \& Q_{02}$ closed and $Q_{1} \& Q_{2}$ open), the frequency is configured to be $500 \mathrm{~Hz}$ where the frequency dependent effect is negligible. The amplitude of $V_{1}$ is adjusted to generate a $B-H$ loop with peak field strength $\hat{H}=20 A / m$, as shown in Fig. 4a. Secondly, the test setup is reconfigured to provide $50 \mathrm{kHz}$ symmetrical PWM excitation without zero-voltage period, where the switches $Q_{01} \& Q_{02}$ are open, $Q_{1} \& Q_{2}$ actively switched. The output of the voltage sources $V_{3}$ and $V_{4}$ are provided by the reference signal with the same amplitude but opposite polarity, which is adjusted to generate a $B-H$ loop with peak field strength $\hat{H}=20 A / m$, as shown in Fig. 4b ( $V_{1}$ is tuned to ensure zero DC bias). Comparing Fig. 4a and Fig. 4b, only slight shape difference can be detected, while the per-cycle energy loss (indicated by the loop area) exhibits less than $10 \%$ difference. This indicates that the part of frequency independent core loss still dominates in this operating condition, although the switching frequency $50 \mathrm{kHz}$ is significantly higher than $500 \mathrm{~Hz}$.

Afterwards, a zero voltage period of duration $t_{0}=8 \mu \mathrm{s}$ is inserted to the PWM excitation with the mid-point switches $Q_{01} \& Q_{02}$ activated, the voltage output of $V_{3}$ and $V_{4}$ are adjusted to ensure that the peak amplitude is still equal to $\hat{H}=20 A / m$. The measured $B-H$ characteristic and time domain primary current and secondary voltage are shown in Fig. 5a and Fig. 5b, respectively. In comparison to Fig. 4, significant larger $B-H$ loop area can be observed especially in the location approaching the peak. The flat part between the points "a" and "b" arise from the relaxation effect, where the field strength decreases although nearly zero excitation is applied, as has also been observed in the work of [4]. According to the observed phenomenon, the basic model structure of relaxation effect is derived using permeancecapacitor based magnetic circuit in the next section.

\section{Discussion of measurement accuracy}

During measurement and data post processing, one should pay attention to the influence of the phase error between the current and voltage. Authors of [16] has explored that the percentage error of the core loss power can be expressed as:

$$
\Delta_{\%}=\tan \left(\Phi_{\mathrm{v}-\mathrm{i}}\right) \cdot \Delta \Phi
$$

where $\Phi_{\mathrm{v}-\mathrm{i}}$ is the absolute phase shift between the measured primary winding current and second winding voltage under sinusoidal excitation, while $\Delta \Phi$ is the error of the phase shift arising from the measurement system. Equation (1) indicates that, the closer to $90^{\circ}$ is $\Phi_{\mathrm{v}-\mathrm{i}}$, the more sensitive is the percentage error to $\Delta \Phi$. Different modifications on the measurement circuit structure has been proposed by the work of [17], [18] and [19], so as to drag $\Phi_{\mathrm{v}-\mathrm{i}}$ away from $90^{\circ}$ and thus decrease the sensitivity to $\Delta \Phi$. There high excitation frequency of several or tens of $\mathrm{MHz}$ was discussed, the frequency in this work $(50 \mathrm{kHz})$ however, is far below that range. Considering the fact that $\Delta \Phi$ is proportional to the excitation frequency [20], the influence of $\Delta \Phi$ is significantly lower in the frequency range of this work. Moreover, with the modification although the core loss power can be more accurately measured, the exact shape of the $B-H$ is not available anymore, which however, is necessary for the model parametrization and verification in this work. Therefore the original circuit structure from [16] is still adopted.

The influence of phase error in the measurement of low frequency $(500 \mathrm{~Hz})$ sinusoidal excitation can be neglected. For the case of $50 \mathrm{kHz}$ PWM excitation, phase error compensation has been applied in post processing. Phase error may exist in both voltage and current measurements:

- Voltage measurement: In the $50 \mathrm{kHz}$ measurement, due to the sufficiently large amplitude of the excitation voltage, the amplification stage is bypassed. The secondary winding is directly connected to the oscilloscope via shielded axial cable ( $V_{\mathrm{s}}$ in Fig. 6), which has the same length as that of the current measurement, so that the phase delay of the voltage measurement can be neglected.

- Current measurement: According to the datasheet of the ferrite material N87 [21], the per-volume core loss $\left(25 \mathrm{C}^{\circ}\right)$ at frequency $f=50 \mathrm{kHz}$ and peak-to-peak flux density $\Delta B=200 \mathrm{mT}$ (amplitude $100 \mathrm{mT}$ ) is $54 \mathrm{~kW} / \mathrm{m}^{2}$, which corresponds to $P_{\text {loss }}=0.08 \mathrm{~W}$ loss power for the core "R $20.0 \times 10.0 \times 7.00$ ", if multiplied by the core volume. The equivalent phase shift between voltage and current can be calculated as: [20]

$$
\Phi_{\mathrm{v}-\mathrm{i}}=\arctan \left(\frac{\left(N_{1} \cdot A \cdot 2 \pi f \cdot \Delta B\right)^{2}}{8 \cdot P_{\text {loss }}} \cdot \frac{1}{2 \pi f \cdot L}\right)
$$

The equation above $\Phi_{\mathrm{v}-\mathrm{i}}=84.6^{\circ}$, where $N_{1}=5$ is the primary winding turns number, $A$ is the cross section area of the core and $L$ the equivalent inductance calcu- 
lated from the $A_{L}$ value provided by the manufacturer's datasheet [21].

If we target on $\epsilon_{p h}= \pm 5 \%$ phase error in equation (1), the allowed uncompensated phase error is $\pm 0.272^{\circ}$ at $50 \mathrm{kHz}$. The first source of the uncompensated phase error is the sample step size of the oscilloscope, which is in our case equal to $10 \mathrm{~ns}\left(0.18^{\circ}\right.$ for $\left.50 \mathrm{kHz}\right)$. The second source of the uncompensated phase error arise from the stray inductance of the shunt resistor. The manufacturer of the $0.1 \Omega$ shunt resistor [22] specifies that the stray inductance is typically $0.5 \mathrm{nH}$, which result in a phase angle of $0.09^{\circ}$ at $50 \mathrm{kHz}$. The sum of the phase error of the oscilloscope and shunt resistor is within the criteria $0.272^{\circ}$.

The differential amplifier aims to filter out EMC noise and eliminate the DC voltage bias of the power amplifier $V_{1}$, the phase delay between the voltage drop on the shunt resistor and the differential amplifier's output can be explicitly compensated: As demonstrated in the simplified circuit schematic shown in Fig. 6, for each measurement, the voltage $V_{\mathrm{sh}}$ between the positive terminal of the shunt and ground (with DC bias of $V_{1}$ ) is connected to the oscilloscope using a coaxial cable with the same length as the differential amplifier's output $V_{\text {op }}$ (without DC bias of $V_{1}$ ). At the moment when the excitation voltage becomes positive from zero, the time difference between the turning point of $V_{\mathrm{sh}}$ and that of $V_{\mathrm{op}}$ is considered as the phase delay $\Delta \Phi$ to be compensated (with the uncompensated phase error discussed before). During the data post processing to calculate core loss, the current measurement should be shifted by $-\Delta \Phi$. For the measurement of other materials and cores, compensation is applied following similar consideration.

Beside the phase error, the equipment accuracy also add up to the measurement error, which can be found in the datasheet of the corresponding components and devices. The major contribution comes from:

- The shunt resistor has an error of $\epsilon_{s h}= \pm 0.5 \%$.

- The chip resistor to configure the gain of the differential amplifier has $\epsilon_{a m p}=0.1 \%$ error.

- The oscilloscope has a measurement error of $\epsilon_{o s c}=$ $\pm 2 \%$.

Combining these three error sources and the phase error together, the system measurement error is calculated by

$$
\epsilon_{s y s}=\left(1+\epsilon_{s h}\right) \cdot\left(1+\epsilon_{a m p}\right) \cdot\left(1+\epsilon_{o s c}\right) \cdot\left(1+\epsilon_{p h}\right)-1
$$

The equation above yields $\epsilon_{\text {sys }}= \pm 7.7 \%$.

\section{PROPOSED MODEL}

\section{A. Model behaviour}

The model for frequency independent hysteresis effect introduced in [10] is taken as starting point for the modeling approach in this work. The equivalent electric-magnetic circuit of the test setup is demonstrated in Fig. 7a, which includes the PWM excitation represented by a voltage source, the winding block as interface to magnetic circuit (Fig. 7b) and the permeance building block modeling the core sample. The internal structure of the permeance block, which is able to represent a complete magnetic core of simple geometry or one part of that with complex geometry, is demonstrated in Fig. 7c. Please note that in the work of [10] two variable permeance blocks accounting for irreversible- and reversible magnetization are connected in parallel for illustration purpose, while in this work they are structurally merged into one and the differential permeability of the irreversible- and reversible components are summed up as $\mathscr{P}_{1}(H)$, which is physically equivalent to that in [10].

After the parameters of the formula in calculating $\mathscr{P}_{1}(H)$ are completely identified using the experimental measurement at low frequency sinusoidal excitation, as has been described by [10], the relaxation effect can be incorporated. As illustrated in Fig. 8, at first, the permeance block $\mathscr{P}_{1}$ is split into two permeance blocks in parallel, namely $\mathscr{P}_{1}^{\prime}$ and $\mathscr{P}_{2}$. It is firstly assumed that the permeance $\mathscr{P}_{2}$ is constant.

$$
\mathscr{P}_{1}^{\prime}(H)=\mathscr{P}_{1}(H)-\mathscr{P}_{2}
$$

Secondly a "magnetic" resistor $R_{m}$ is connected to the branch of $\mathscr{P}_{2}$. Similar to the behavior of a electrical resistor, the "magnetic" voltage drop (magneto-motive force MMF $F$ ) across $R_{m}$ is equal to $R_{m}$ multiplied by the "magnetic" current (flux changing rate $\dot{\Phi}=d \Phi / d t$ ), governed by the equation:

$$
F=R_{m} \cdot \dot{\Phi}
$$

Please note that there is no magnetic resistor placed at the left hand side of the hysteresis permeance, which was present in the structure proposed by [23] mainly accounting for eddy current effect. This complies to the fact that we neglect the

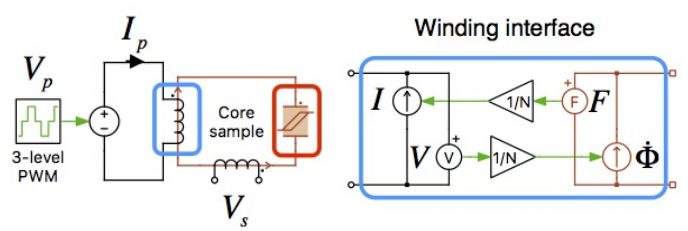

(a)

(b)

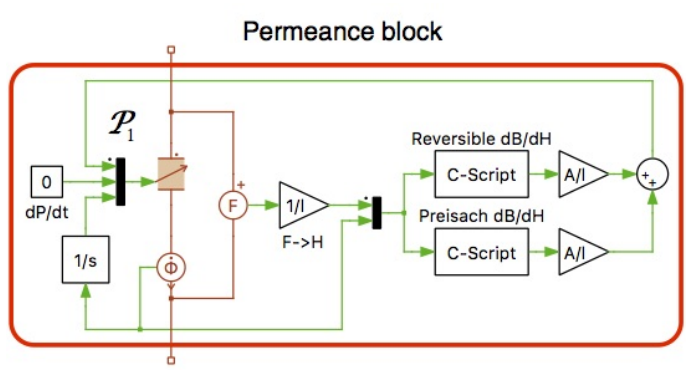

(c)

Fig. 7: (a) Simplified model of the test setup including both electrical- and magnetic circuit; (b) Internal structure of the permeance block; (c) Winding interface using gyrator structure. 


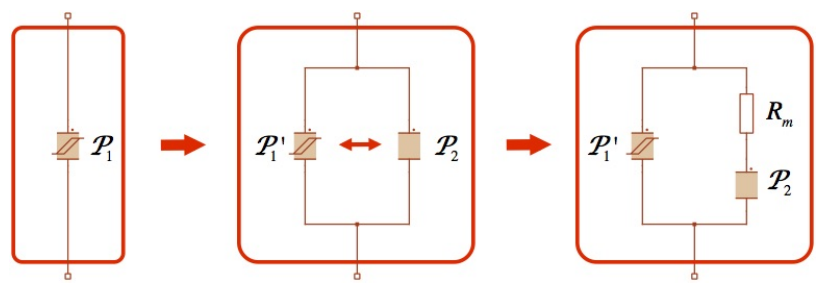

Fig. 8: Modification of the original hysteresis permeance block to incorporate relaxation effect, where the permeance value of $\mathscr{P}_{1}^{\prime}$ is provided by subtracting $\mathscr{P}_{2}$ from $\mathscr{P}_{1}$.

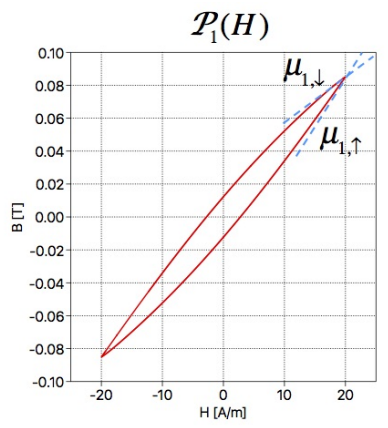

II
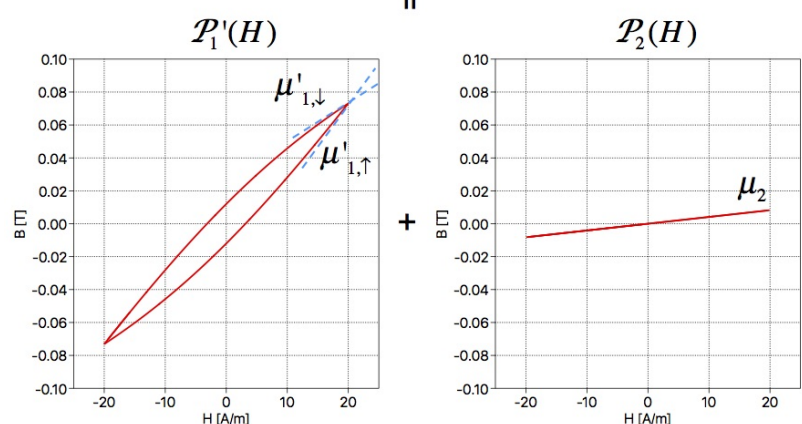

Fig. 9: Summation of the $B-H$ characteristic of $P_{1}^{\prime}$ and $P_{2}$ under low frequency excitation.

eddy current effect of ferrite materials, which has negligible contribution to the core loss, compared to the relaxation effect and frequency independent hysteresis. It is also to be emphasized that if sufficiently low frequency sinusoidal excitation is applied, $R_{m}$ has negligible effect and the $B-H$ characteristic of the overall system is equal to that of the original $\mathscr{P}_{1}$ (since the summation of $\mathscr{P}_{1}^{\prime}$ and $\mathscr{P}_{2}$ is equal to $\left.\mathscr{P}_{1}\right)$, as the symmetrical loops within the range $[-\hat{H},+\hat{H}]$ in Fig. 9 demonstrated. If the excitation is high frequency PWM especially with zero voltage period, the relaxation effect taking place in reality can be covered by $R_{m}$. In the next step, the mechanism of relaxation effect is interpreted in the magnetic circuit. We still take the simplified circuit model of the test setup in Fig. 7a as example, with the original permeance block proposed in [10] extended to the new structure. The circuit resistance and winding resistance are neglected. The time area in which the external excitation (generated via switching the T-shape bridge) varies from positive to negative (including the zero voltage period in between) is analyzed, the DC voltages of $V_{3}$ and $V_{4}$ are assumed to be tuned (equal to $\pm V_{i n}$ ) so that the

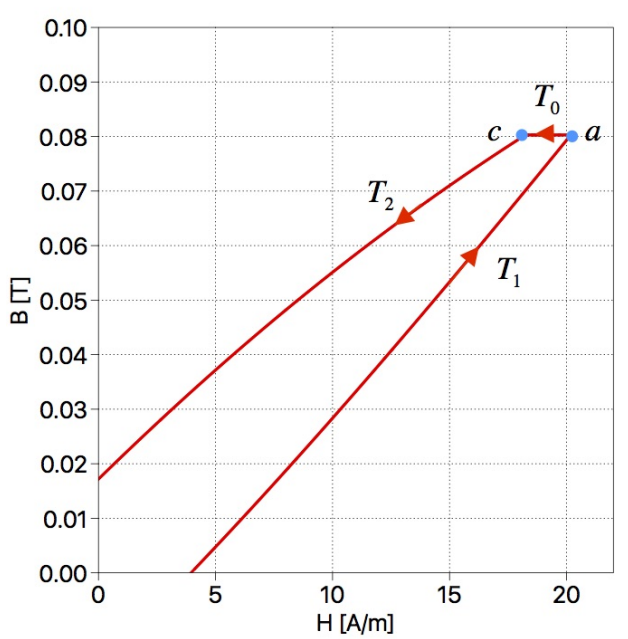

(a)
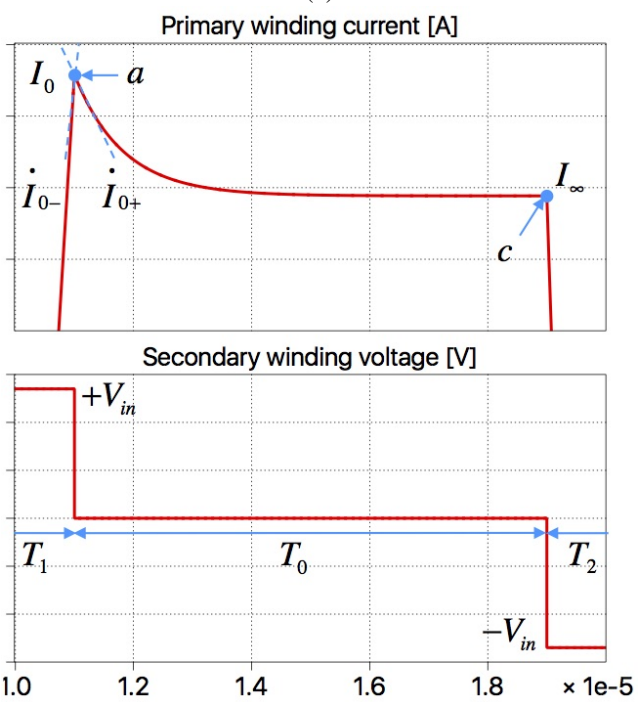

(b)

Fig. 10: Circuit behavior of the proposed model in reproducing relaxation effect (a) B-H characteristic including a flat part near the peak (from point $a$ to $c$ ). (b) Time domain waveform with current descending rapidly at the beginning of the zerovoltage period.

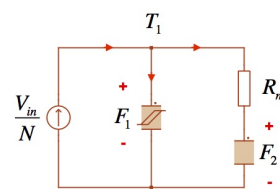

(a)

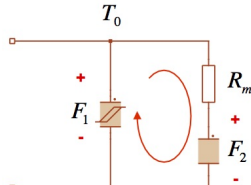

(b)

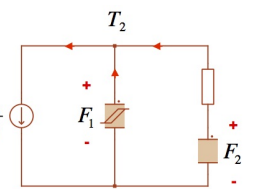

(c)
Fig. 11: Equivalent circuit configuration (a) Pos. excitation; (b) Zero voltage period; (c) Neg. excitation.

equivalent field strength is controlled in the range $[-\hat{H},+\hat{H}]$ :

- Period $T_{1}$ : When the switch $Q_{1}$ is turned on, positive voltage $+V_{i n}$ is applied on the primary winding and the circuit current rises, as the interval $T_{1}$ in Fig. 10b demonstrates. In the permeance-capacitor based magnetic 
circuit, the voltage excitation is translated as a "magnetic" current source (flux rate $\dot{\Phi}=V_{i n} / N$ ), which charges the two permeance-capacitors $\mathscr{P}_{1}^{\prime}$ and $\mathscr{P}_{2}$, as demonstrated in Fig. 11a. The "magnetic" voltage (magneto-motiveforce MMF, denoted as $F_{1}$ ) of permeance $\mathscr{P}_{1}^{\prime}$ increases, which leads to increased equivalent field strength, as shown in Fig. 10b (rising interval $T_{1}$ ). The equivalent field strength looking from the excitation source is given by:

$$
H=F_{1} / l
$$

where $l$ is the equivalent magnetic path length of the core. Under positive excitation, the MMF of $\mathscr{P}_{2}$ (denoted as $F_{2}$ ) also increases, however in a slower rate due to the existence of the resistor $R_{m}$. This leads to the fact that at the end of period $T_{1}$ (point $a$ in Fig. 10a), $F_{2,0}$ is lower than $F_{1,0}$. The rising rate $\dot{F}_{1,0-}=d F_{1} / d t\left(0_{-}\right)$directly before point $a$ is given as

$$
\dot{F}_{1,0-}=\frac{l}{\left(\mu_{1, \uparrow}-\mu_{2}\right) \cdot A} \cdot\left(\frac{V_{i n}}{N}-\frac{F_{1,0}-F_{2,0}}{R_{m}}\right)
$$

In the equation above, $A$ is the cross section area of the core sample and $N$ the primary winding's turns number. $\mu_{1, \uparrow}$ is the ascending permeability of the original frequency-independent model at the peak $H=\hat{H}$ (Fig. 9). The peak MMF $\left(F_{1,0}\right)$ and the rising rate $\left(\dot{F}_{1,0-}\right)$ can be determined from the peak primary winding current $I_{0}$ and its rising rate $\dot{I}_{0-}$ using equation below.

$$
F_{1,0}=I_{0} \cdot \frac{l}{N}, \quad \dot{F}_{1,0-}=\dot{I}_{0-} \cdot \frac{l}{N}
$$

- Period $T_{0}$ : After the switch $Q_{1}$ is turned off and $Q_{01} \& Q_{02}$ turned on, zero voltage is applied on the primary winding. In the magnetic circuit, this situation corresponds to a open circuit on the winding side, as demonstrated in Fig. 11b. Starting from point $a$ on, there is only a circulating "magnetic" current (flux rate) flowing in the loop composed of $\mathscr{P}_{1}^{\prime}$ and $\mathscr{P}_{2}$. Due to the higher initial MMF on $\mathscr{P}_{1}^{\prime}$, the circulating "magnetic" current charges $\mathscr{P}_{2}$ and discharges $\mathscr{P}_{1}^{\prime}$, the initial value of the MMF's falling rate immediately after the zero-voltage period starts is given by:

$$
\dot{F}_{1,0+}=\dot{I}_{0+} \cdot \frac{l}{N}=\frac{l}{\left(\mu_{1, \downarrow}-\mu_{2}\right) \cdot A} \cdot \frac{F_{2,0}-F_{1,0}}{R_{m}}
$$

$\mu_{1, \downarrow}$ is the descending permeability of the original frequency-independent model at the peak $H=\hat{H}$ (Fig. 9). Afterwards, on one hand, $\mathscr{P}_{1}^{\prime}$ is discharged and $F_{1}$ falls down, which is reflected by the decreasing equivalent field strength in Fig. 10a (from point $a$ to $c$ ). Since the flux rate out of the winding side is zero, the equivalent flux density remains unchanged. On the other hand, $\mathscr{P}_{2}$ is charged up, and the MMF of both permeances gradually approach a common value. This behavior complies to the explanation of relaxation effect given in [1], that a new thermal equilibrium is gradually achieved where the system has minimum magnetic energy state (the magnetic energy stored in the permeance capacitors is reflected by the MMF). Please note that after the MMF of the two permeances are balanced, the total energy stored is lower than that at the beginning of the zero-voltage period, and the energy difference is actually burned by the magnetic resistor $R m$, which generates extra core loss. After sufficiently long time (e.g. point $c$ in Fig. 10), following similar law of series connected electrical capacitors, the balanced MMF $F_{\infty}$ fullfills the relation in below:

$$
\frac{\mu_{2} \cdot A}{l} \cdot\left(F_{2,0}-F_{1,0}\right)=\frac{\mu_{1, \downarrow} \cdot A}{l} \cdot\left(F_{\infty}-F_{1,0}\right)
$$

$F_{\infty}$ can be derived from the stabilized current $I_{\infty}$ (denoted in Fig. 10b) as $F_{\infty}=I_{\infty} \cdot N / l$.

- Period $T_{2}$ : Negative voltage appears on the primary winding when the switch $Q_{2}$ is turned on at point $c$ in Fig. 10b, which corresponds to a flux rate source in the magnetic circuit flowing in opposite direction compared to that in the period $T_{1}$, as demonstrated in Fig. 11c. From then on, both field strength and flux density decreases rapidly as shown in Fig. 10a. Also that after this negative voltage period there is another zero-voltage interval $T_{0}$ coming and the situation is similar as discussed before, however with the relaxation effect observable at the negative peak $-\hat{H}$.

\section{B. Parameter identification}

Up to this stage, if we substitute the slopes $\dot{I}_{0-}, \dot{I}_{0-}$ and the peak current $I_{0}$, the stabilized current $I_{\infty}$ by the corresponding values from the measurement results (e.g. that in Fig. 5b), as well as the permeability $\mu_{1, \downarrow}$ and $\mu_{1, \uparrow}$ by that measured under low frequency sinusoidal excitation (with the same peak field strength), the equations (7), (9) and (10) can be solved for $R_{m}$ and $\mu_{2}$ and $F_{2,0}$. Thus all parameters of the extended model can be explicitly determined. With $\mu_{2}$ and the geometry of the core sample, $\mathscr{P}_{2}$ is calculated as

$$
\mathscr{P}_{2}=\frac{\mu_{2} \cdot A}{l}
$$

The measurement results for parameter identification need to be taken with the highest switching frequency and longest zero-voltage period of concern, where the relaxation effect can be obviously observed. In the next section, it is proved that the parametrized model is able to reproduce the material's behavior at any lower switching frequency and shorter zerovoltage period.

Also please note that in the real test setup, the circuit resistance leads to the fact that the current damped down slowly after the active interval of relaxation effect, rather than stabilized on a final value $I_{\infty}$, as the part between point $b$ and $c$ in Fig. 5b indicates. The time point $b$ when flux starts to decrease can be identified in the measured corresponding $B-H$ plot (in Fig. 5a), which indicates that the active period of relaxation effect is over. In this case, the current at point $b$ should be considered as $I_{\infty}$ for parameter identification. 

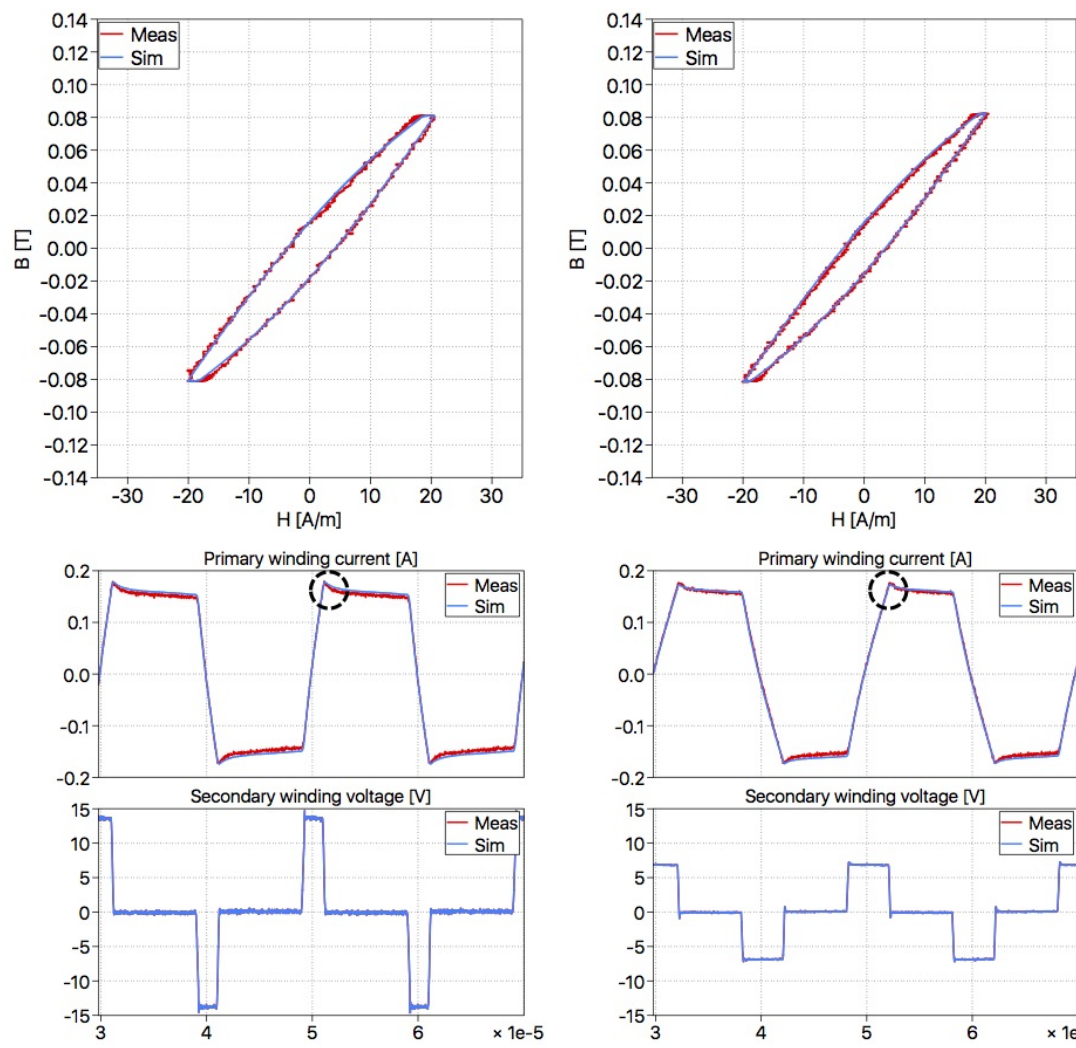

(a)

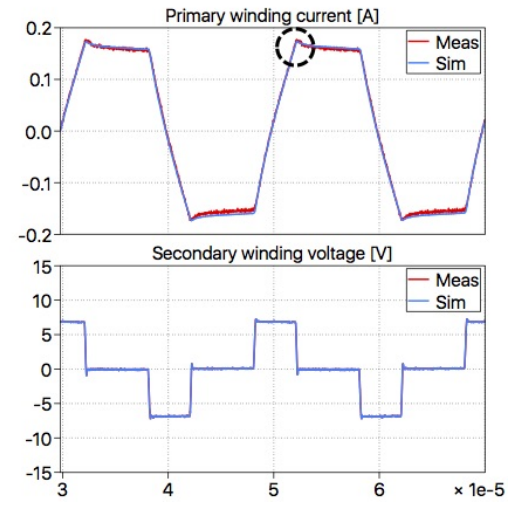

(b)
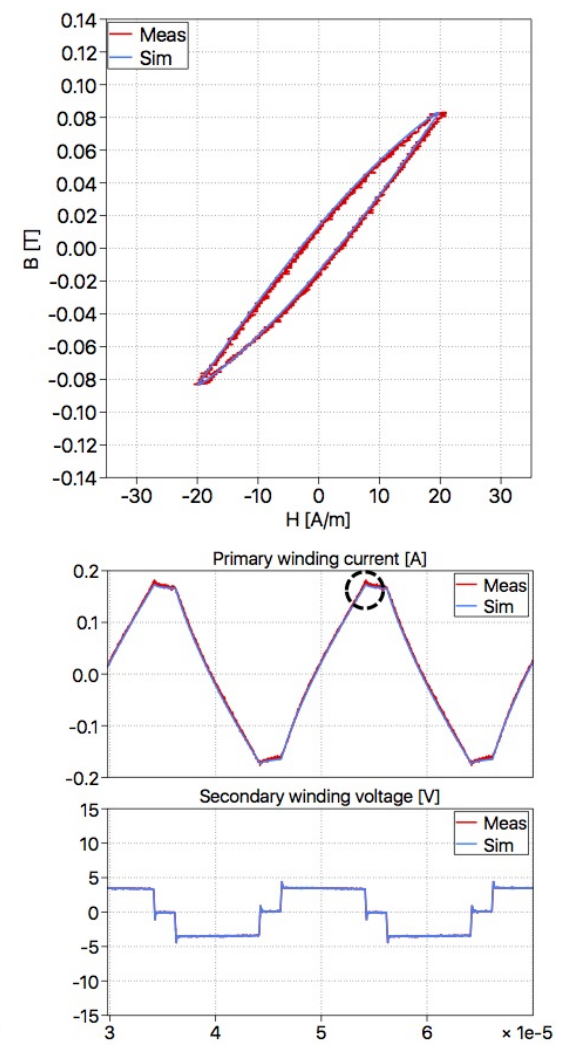

(c)

Fig. 12: $B-H$ characteristic and time domain waveform comparison between measurement and simulation under $50 \mathrm{kHz}$ PWM excitation with peak field strength $\hat{H}=20 \mathrm{~A} / \mathrm{m}$ and different percentage of $T_{0}$ (a) $80 \%$; (b) $60 \%$; (c) $20 \%$.

\section{EXPERIMENTAL VERIFICATION}

In this section, several verification schemes of the proposed model are carried out using the test setup introduced in section II. The ferrite material N87 is considered as the first study case with the toroidal core of shape code "R $20.0 \times 10.0 \times 7.00$ " as sample. The model is parametrized following the process from section III-B, which is composed of two steps:

- Parameters of frequency independent effect (model proposed in [10]) are identified using experimental measurement under sinusoidal excitation of $500 \mathrm{~Hz}$, where frequency dependent effect is negligible.

- Parameters of relaxation effect $\left(\mathscr{P}_{2}\right.$ and $\left.R_{m}\right)$ are identified using experimental measurement under $50 \mathrm{kHz}$ PWM excitation with $80 \%$ zero-voltage period, where the amplitudes of the DC sources are trimmed to obtain peak field strength amplitude equal to $\hat{H}=20 \mathrm{~A} / \mathrm{m}$. The resulted parameters are $\mathscr{P}_{2}=3.18 \cdot 10^{-7} \mathrm{H}$ and $R_{m}=3.03 \Omega^{-1}$

The circuit model is established in system level simulation software PLECS complying to the test setup, with the same structure shown in Fig. 7a. In order to exclude the influence of the circuit resistance of the test setup, the equivalent voltage source connected to the primary winding is fed with the measured secondary voltage multiplied by $N_{1} / N_{2}$, where
$N_{1}=N_{2}=5$ are the turns number of the primary- and secondary winding, respectively.

\section{A. Verification under PWM excitation with different zero- voltage period}

In the first group of verification schemes, the fidelity of the proposed model is verified under $f_{s w}=50 k \mathrm{~Hz}$ PWM excitation with different percentage of zero-voltage period $(80 \%, 60 \%, 20 \%)$, while the DC voltage sources are adjusted to maintain the equivalent peak field strength $\hat{H}=20 \mathrm{~A} / \mathrm{m}$. As demonstrated in Fig. 12, with decreasing percentage of zero-voltage period, the flat part near the peak of the $B-H$ loops becomes narrower, which result in decreasing per-cycle energy loss (loop area) and indicates that the contribution from relaxation effect reduces. The proposed model is able to reproduce the hysteresis loop with good accuracy, and the corresponding core loss power are compared to measurement in Fig. 13, the error turns out to be under $5 \%$. The presence of relaxation effect is also clearly reflected on the time domain waveform, where the primary winding current sinks with different steepness immediately after the zero-voltage period begins, as has been highlighted by dashed circles. In the simulation model, the frequency independent core loss (magnetic hysteresis loss) can be easily extracted by multiplying the $F$ and $\dot{\Phi}$ of the permeance $\mathscr{P}_{1}^{\prime}$, integrating over one switching period and scaled by the switching frequency: 


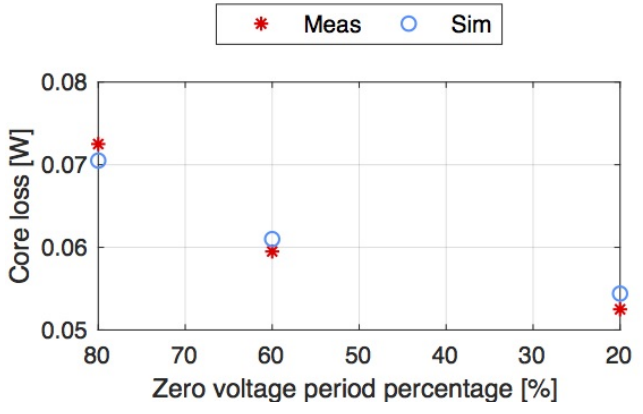

Fig. 13: Core loss comparison between measurement and simulation under $50 \mathrm{kHz}$ PWM excitation with peak field strength $\hat{H}=20 \mathrm{~A} / \mathrm{m}$ and different percentage of $T_{0}$.

$$
P_{\text {loss }, \text { hyst }}=f_{s w} \cdot \int_{T} F \cdot \dot{\Phi} \cdot d t
$$

Subtracting the frequency independent part from the simulated total loss, the frequency dependent part due to relaxation effect can be directly obtained, as demonstrated in TABLE I. With decreasing percentage of the zero-voltage period, the frequency dependent core loss reduces, while the frequency independent one remains the same.

TABLE I: Loss splitting of the simulation model into frequency independent- and dependent parts $[W]$

\begin{tabular}{c|c|c|c|}
\hline$T_{0}$ in percentage & $80 \%$ & $60 \%$ & $20 \%$ \\
\hline Total loss & 0.071 & 0.061 & 0.054 \\
\hline$f_{s w}$ independent loss & 0.045 & 0.045 & 0.045 \\
\hline$f_{s w}$ dependent loss & 0.026 & 0.016 & 0.009 \\
\hline
\end{tabular}

Afterwards, the frequency of the PWM excitation is reduced to $25 \mathrm{kHz}$, while the DC voltage sources are configured so that the equivalent peak field strength remains at $\hat{H}=20 \mathrm{~A} / \mathrm{m}$. The core loss powers from the same model with $90 \%, 60 \%$ and $20 \%$ zero-voltage period (longer than that in $50 \mathrm{kHz}$ case) are compared to the measurement in Fig. 14. Good match is achieved here as well. One should notice that the hysteresis loop under $25 \mathrm{kHz}$ excitation with $90 \%$ zero-voltage period has almost the same shape as that under $50 \mathrm{kHz}$ excitation

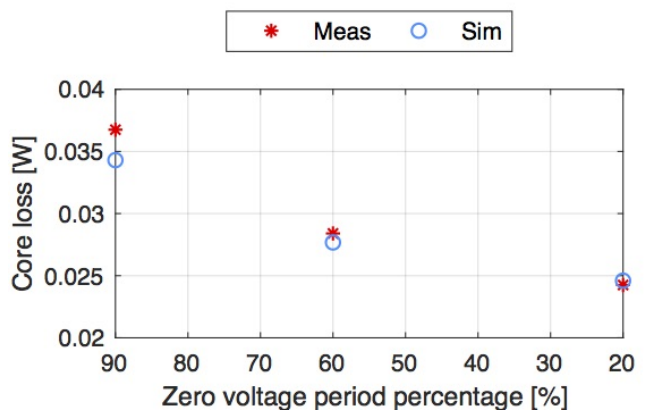

Fig. 14: Core loss comparison between measurement and simulation under $25 \mathrm{kHz}$ PWM excitation with peak field strength $\hat{H}=20 \mathrm{~A} / \mathrm{m}$ and different percentage of $T_{0}$. with $80 \%$ zero-voltage period, and the core loss power of the later is two times of the former. This relation indicates that the per-cycle energy loss are the same, which has also been observed by the authors of [4]. This can be ascribed to the fact that the amplitude and duration of the non-zero (positive or negative) voltage excitation are the same in these two cases, while the zero-voltage phase are both long enough, so that the new thermal equilibrium can be completed reestablished after the relaxation process (permeance capacitors fully recharged) and the same amount of excessive energy loss is burned.

\section{B. Verification under PWM excitation with different duty cycle}

In multiple existing publications, significantly larger core loss was reported under strongly asymmetrical PWM excitation than that with $50 \%$ duty-cycle, which can be ascribed to frequency dependent effect of ferrite materials. In the second group of verification schemes, $50 \mathrm{kHz} \mathrm{PWM}$ excitation with different duty cycles $d=10 \%, 20 \%, 50 \%$ (without zerovoltage period) is applied to the same core sample. The DC voltage sources are configured following the constraint $V_{3}=(1-d) \cdot V_{4}$, so that symmetrical hysteresis loop with equivalent peak field strength $\hat{H}=20 \mathrm{~A} / \mathrm{m}$ can be obtained. The $B-H$ characteristic and time domain waveforms are compared between simulation result and experimental measurement in Fig. 16.

In comparison to the result under symmetrical PWM excitation shown in Fig. 16c, the top-right corner of the $B-H$ loop under $10 \%$ duty-cycle PWM in Fig. 16a is obviously blunter, which adds up to the loop area. This phenomenon arises from the fact that the amplitude of the positive voltage $\left(V_{3}\right)$ is significantly higher than that of the negative one $\left(V_{4}\right)$, the situation at the positive-to-negative transition is similar to the positive-to-zero transition under PWM excitation with zero-voltage period, so that relaxation effect is taking place with considerable strength. In the case of symmetrical PWM excitation $(d=50 \%)$ however, the material is demagnetized in the same rate as it was magnetized, so that relaxation effect has nearly no time to reestablish the new thermal equilibrium. Using the magnetic circuit in Fig. 11, this can be intuitively interpreted as: Although $\mathscr{P}_{1}^{\prime}$ is charged to higher $F$ than $\mathscr{P}_{2}$

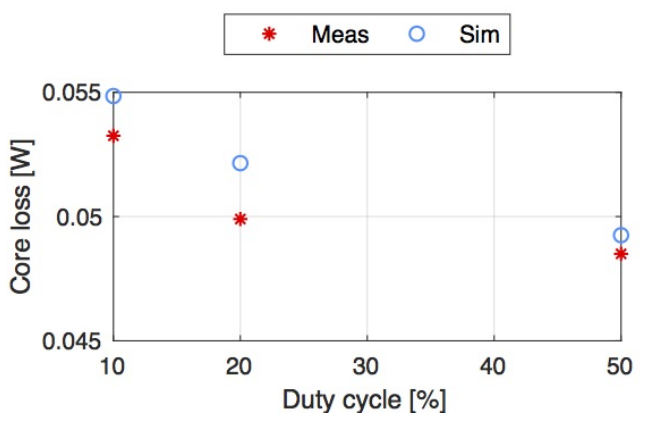

Fig. 15: Core loss comparison between measurement and simulation under $50 \mathrm{kHz}$ PWM excitation with peak field strength $\hat{H}=20 \mathrm{~A} / \mathrm{m}$ and different duty cycle. 

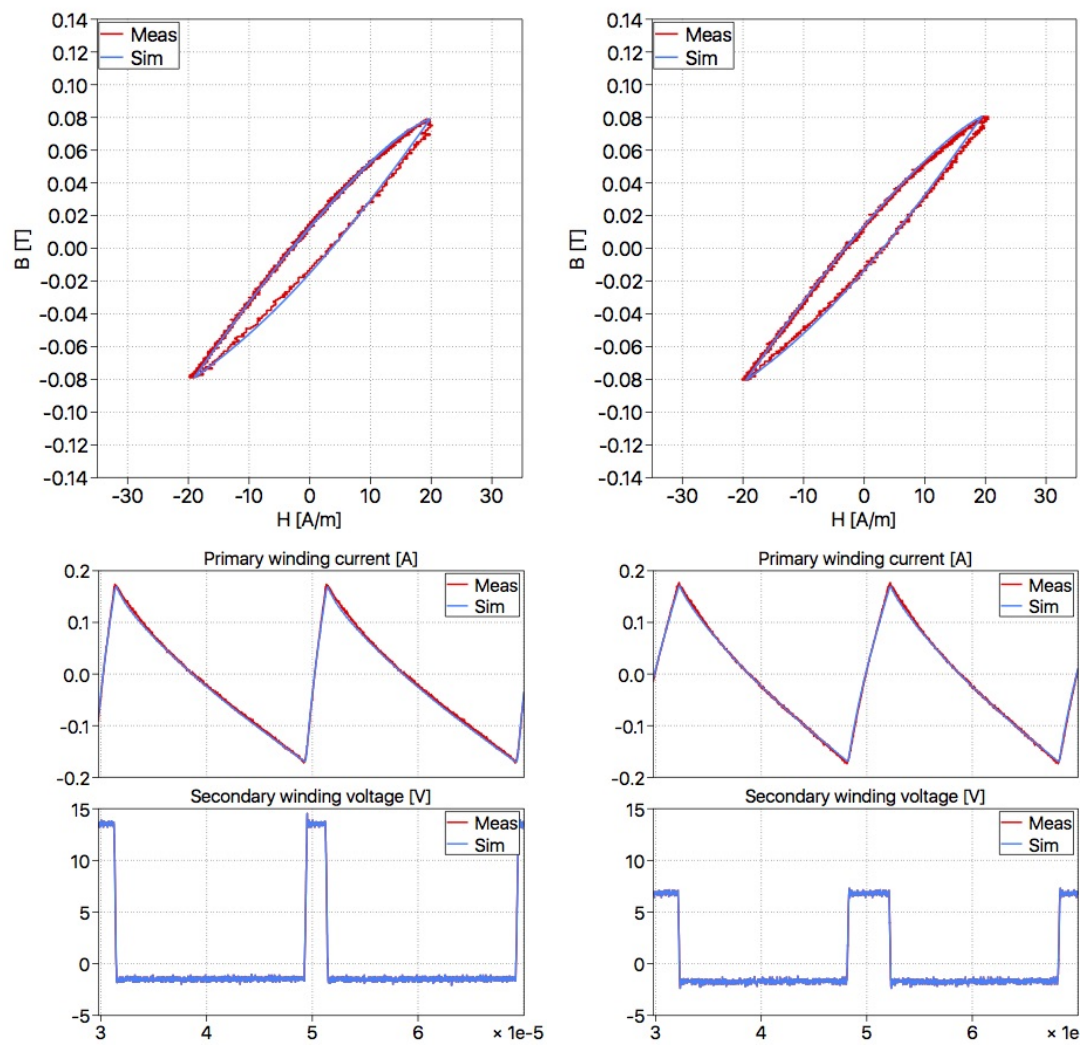

(a)

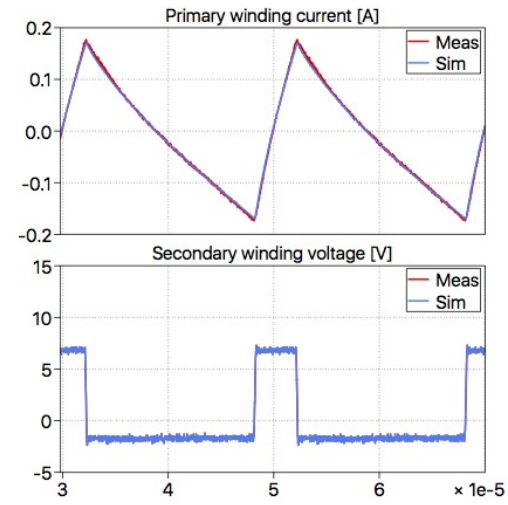

(b)
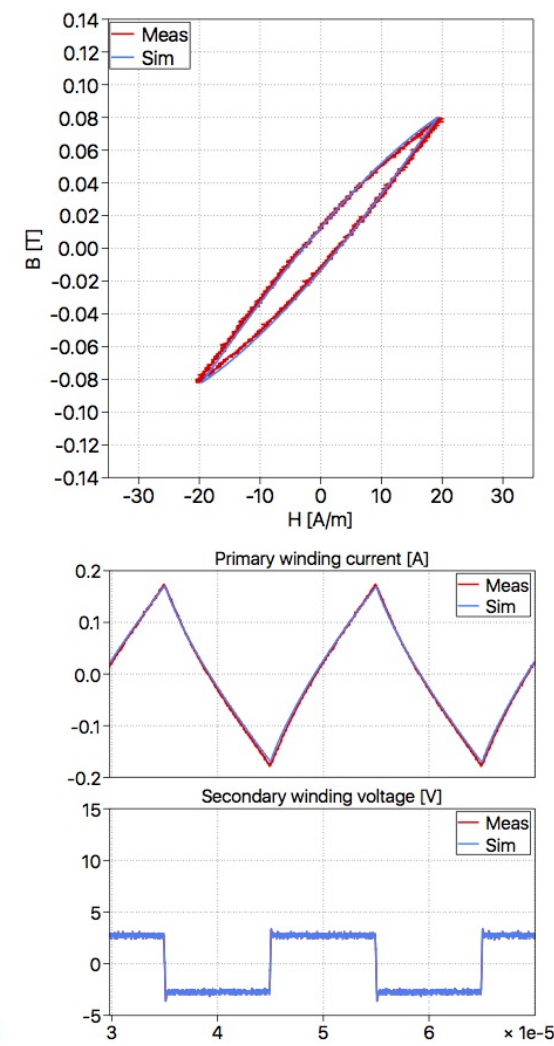

(c)

Fig. 16: $B-H$ characteristic and time domain waveform comparison between measurement and simulation under $50 \mathrm{kHz}$ PWM excitation with peak field strength $\hat{H}=20 A / m$ and different duty cycle (a) $10 \%$; (b) $20 \%$; (c) $50 \%$.

after the positive excitation, it is rapidly discharged afterwards and its $F$ sinks towards that of $\mathscr{P}_{2}$ in such short time that very less energy can be burned on $R_{m}$. All the aforementioned phenomenons have been captured by the proposed model both qualitatively and quantitatively, good match has been obtained in $B-H$ characteristic and time domain waveform shown in Fig. 16 and the core loss shown in Fig. 15.

\section{Verification under PWM excitation with different amplitude}

In the third group of verification schemes, the core sample is still excited by $50 \mathrm{kHz}$ PWM excitation with inclusion of zero-voltage period, the DC voltage sources are configured to generate $B-H$ loops with higher equivalent peak field strength $\hat{H}=30 \mathrm{~A} / \mathrm{m}$. Please note that in this case, the parameters of $\mathscr{P}_{2}$ and $R_{m}$ are re-identified making use of the slopes $\dot{I}_{0-}, \dot{I}_{0-}$ and the currents $I_{0}, I_{\infty}$ measured under $80 \%$ zero-voltage period and the permeability $\mu_{1, \downarrow}$ and $\mu_{1, \uparrow}$ by that measured under low frequency sinusoidal excitation, with equivalent peak field strength $\hat{H}=30 \mathrm{~A} / \mathrm{m}$. The resulted values are $\mathscr{P}_{2}=5.34 \cdot 10^{-7} \mathrm{H}$ and $R_{m}=2.19 \Omega^{-1}$. The simulated $B-H$ loops and time domain waveforms with different zero-voltage period are compared to that from experimental measurements in Fig. 18, and the core loss power are compared in Fig. 17, again the proposed model is able to achieve good accuracy.
In order to accommodate the model to arbitrary amplitude of excitation, $\mathscr{P}_{2}$ and $R_{m}$ need to be realized as piecewise-linear variables with the value on the supporting points identified by $\dot{I}_{0-}, \dot{I}_{0-}, I_{\infty}, \mu_{1, \downarrow}$ and $\mu_{1, \uparrow}$ measured under the corresponding amplitudes of excitation (with sufficiently long zero-voltage period). The modified structure of the model is demonstrated in Fig. 19, where the piecewise-linear relation between the equivalent field strength and the permeance, magnetic resistor values are included in the $f(u)$ function block, with the

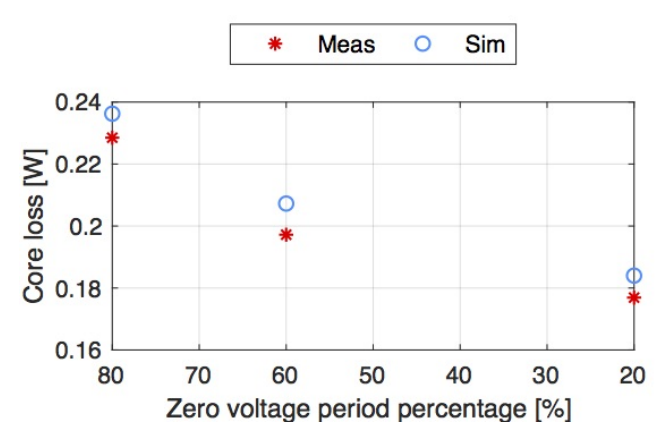

Fig. 17: Core loss comparison between measurement and simulation under $50 \mathrm{kHz}$ PWM excitation with peak field strength $\hat{H}=30 \mathrm{~A} / \mathrm{m}$ and different percentage of zero-voltage period $T_{0}$. 

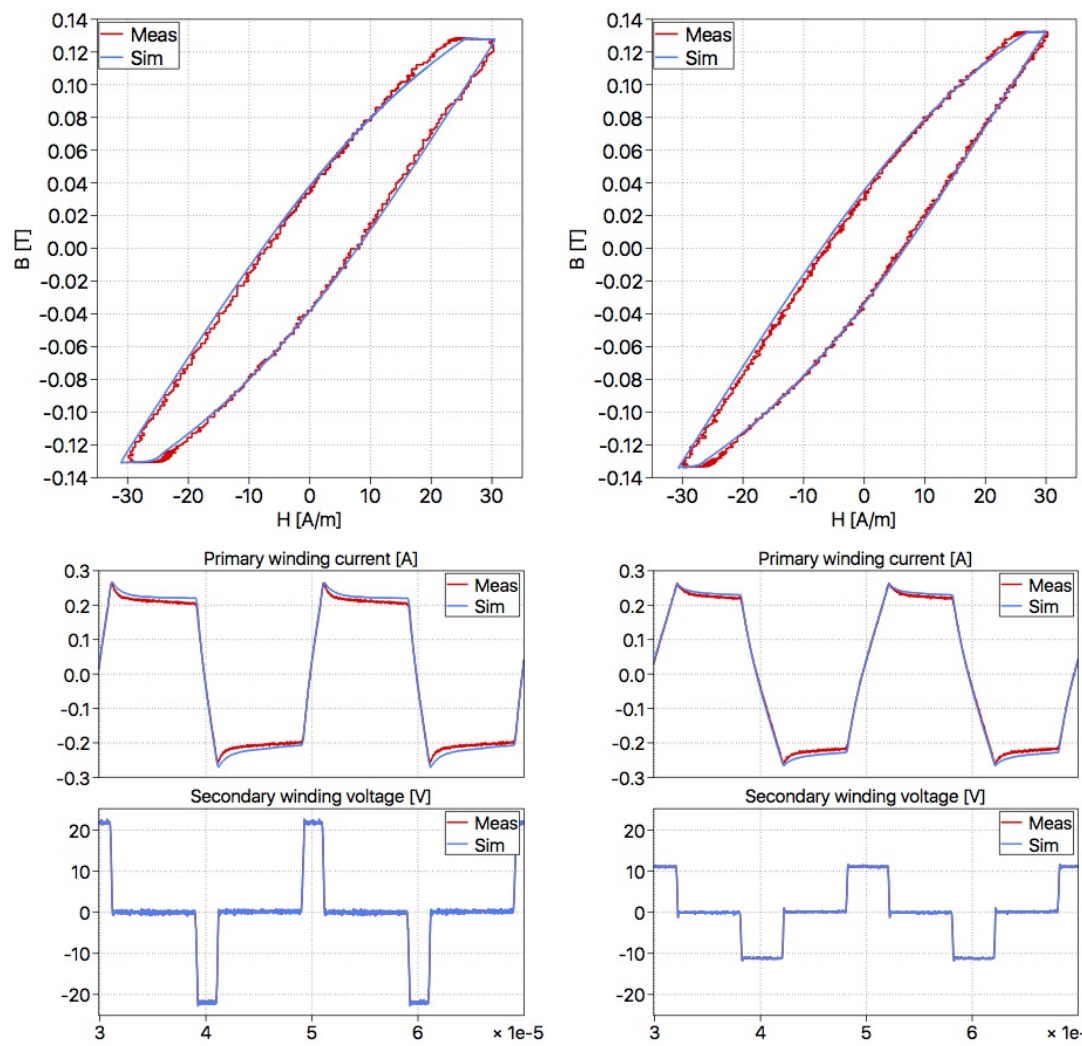

(a)
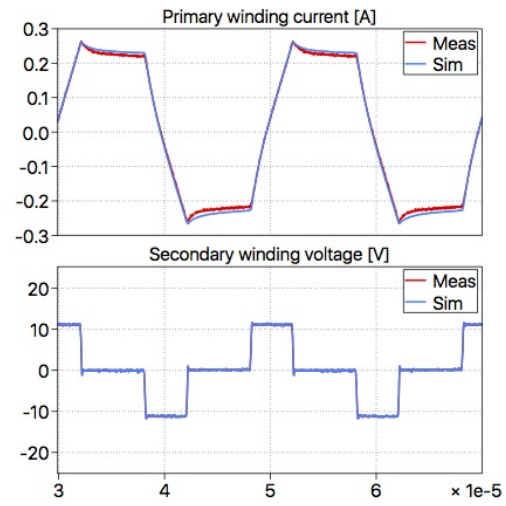

(b)
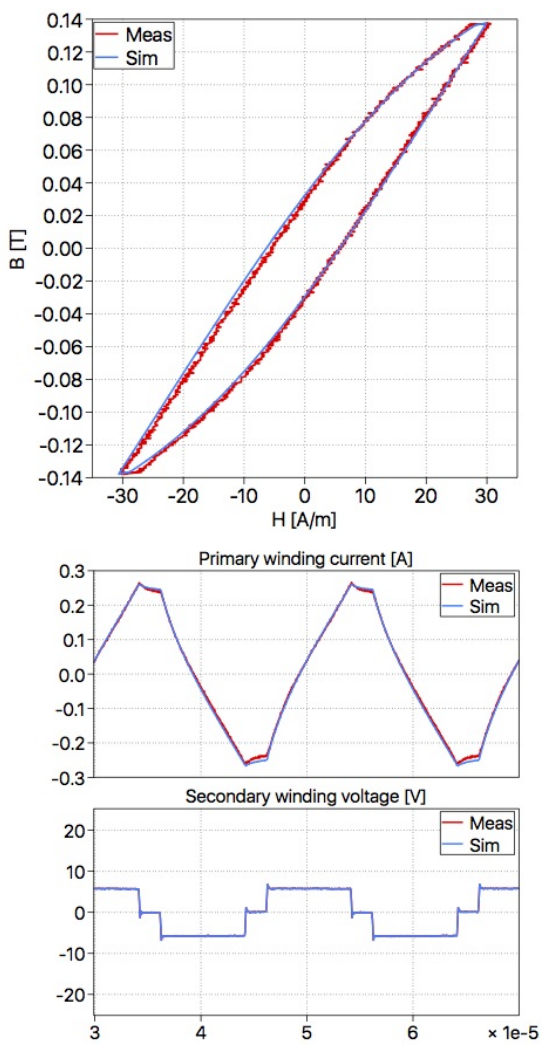

(c)

Fig. 18: $B-H$ characteristic and time domain waveform comparison between measurement and simulation under $50 \mathrm{kHz}$ PWM excitation with peak field strength $\hat{H}=30 A / m$ and different percentage of $T_{0}$ (a) $80 \%$; (b) $60 \%$; (c) $20 \%$.

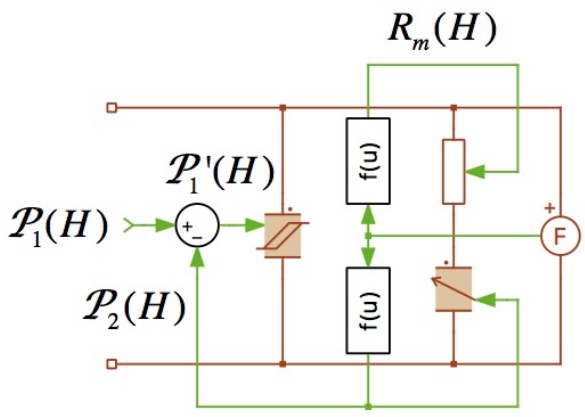

Fig. 19: Piecewise-linear realization of $\mathscr{P}_{2}$ and $R_{m}$ to accommodate arbitrary amplitudes of excitation.

measured MMF of the permeance $\mathscr{P}_{1}^{\prime}$ as input.

\section{Verification under PWM excitation with DC offset}

Using the model structure proposed in Fig. 19, arbitrary hysteresis loop inside the field strength range $H \subset$ $[-30,+30] \mathrm{A} / \mathrm{m}$ can be represented, even with DC bias. For verification, a differential component $\Delta V_{\mathrm{dc}}$ is introduced into the voltage reference of the power amplifiers $V_{3}$ and $V_{4}$, that is:

$$
V_{3}=V_{\mathrm{dc}}+\Delta V_{\mathrm{dc}}, V_{4}=V_{\mathrm{dc}}-\Delta V_{\mathrm{dc}}
$$

Due to the existence of circuit resistance on the primary side, the differential component $\Delta V_{\mathrm{dc}}$ is able to stabilize the hysteresis loop at desired field strength offset. Adjusting both $V_{\mathrm{dc}}$ and $\Delta V_{\mathrm{dc}}$, the biased hysteresis loop in the field strength range $H \subset[0,+30] \mathrm{A} / \mathrm{m}$ is obtained under different percentage of zero-voltage period. The simulation result is compared to the measurement in Fig. 20, where the $B-H$ characteristic exhibits slight asymmetry and is captured by the simulation model. The core loss comparison in Fig. 21 also provides good match. The trend that with shorter zero voltage period, the core loss decreases, is also valid for the biased loop. Observable discrepancy of the loop shape is present in Fig. 20c, where the relaxation effect has minor impact and the frequency independent hysteresis loss dominates, due to the fact that the static hysteresis model is not particularly parametrized taking biased loops into account. Still thanks to the physics-based intrincity of the Preisach model, acceptable accuracy of the biased loop is still achieved. Also please pay attention that the flux density of the biased loop depends on the historical pre-magnetization in reality, which can not be explicitly measured in the existing test setup. Therefore in the $B-H$ plots of Fig. 20 the $B$ range is displayed centered at zero (as integration of the secondary winding voltage). 

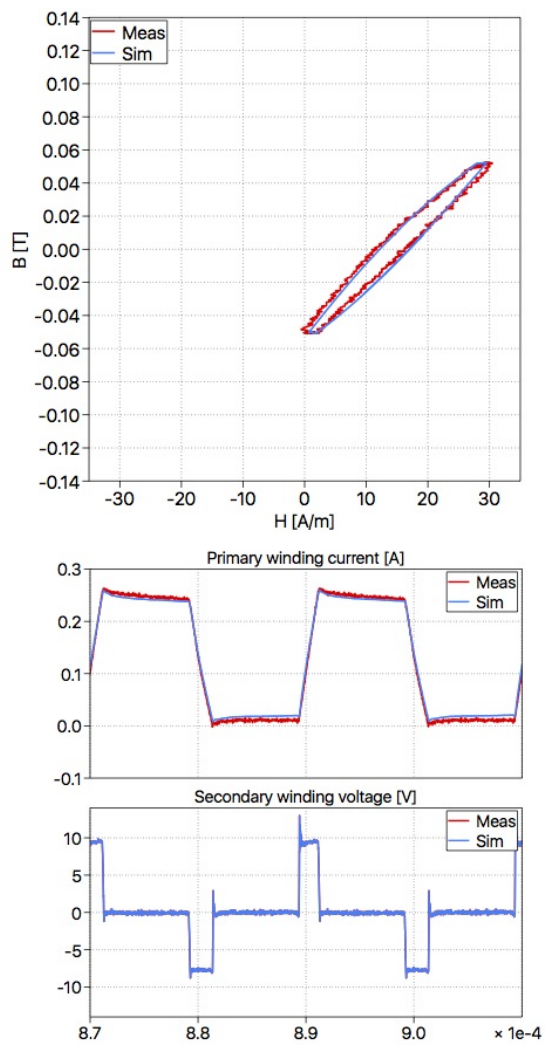

(a)
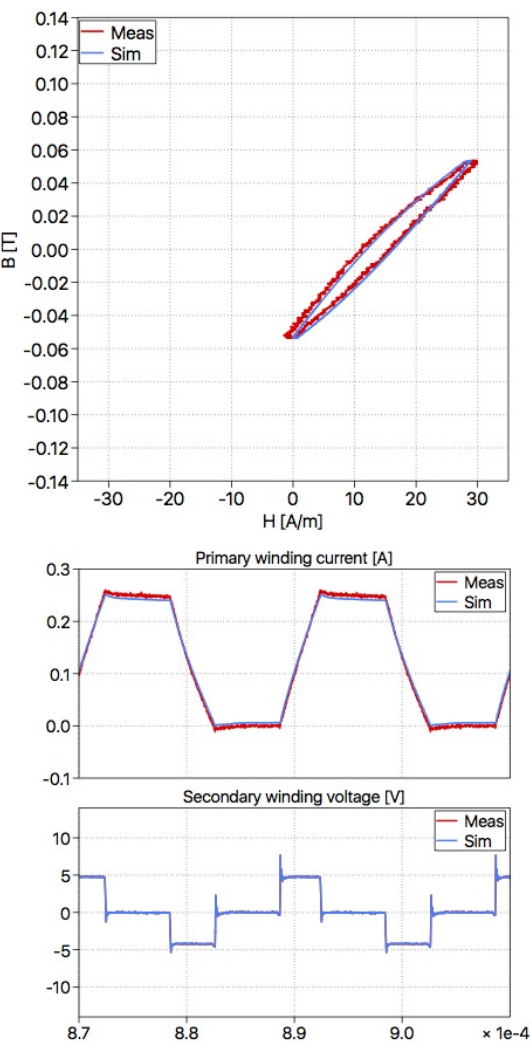

(b)
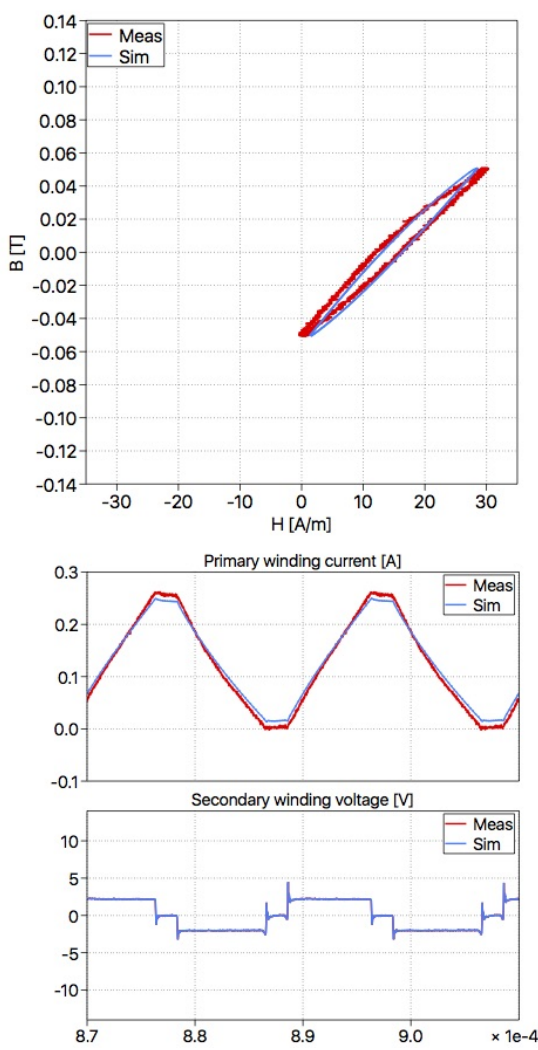

(c)

Fig. 20: $B-H$ characteristic and time domain waveform comparison between measurement and simulation under $50 \mathrm{kHz}$ PWM excitation with biased field strength range $H \subset[0,+30] \mathrm{A} / \mathrm{m}$ and different percentage of $T_{0}$ (a) $80 \%$; (b) $60 \%$; (c) $20 \%$.

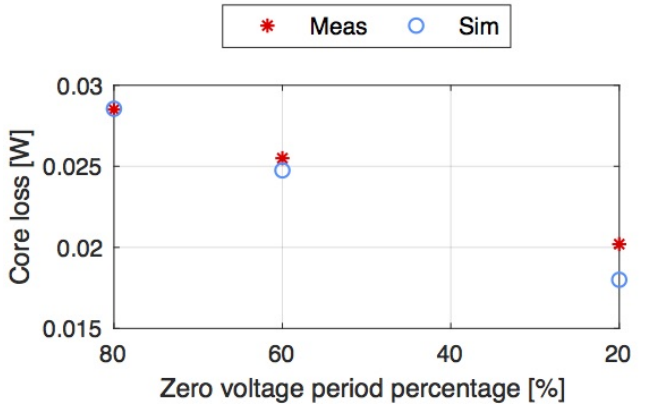

Fig. 21: Core loss comparison between measurement and simulation under $50 \mathrm{kHz}$ PWM excitation with biased field strength range $H \subset[0,+30] \mathrm{A} / \mathrm{m}$ and different percentage of zero-voltage period $T_{0}$.

\section{E. Verification on core sample with different geometries}

Once the parameters of the frequency-independent hysteresis and the frequency-dependent relaxation effect are identified using one core sample for certain ferrite material, the model can be easily extended to other geometries. Assuming that the core sample for parametrization has cross section area $A_{1}$ and equivalent magnetic path length $l_{1}$, the permeance $\mathscr{P}_{1,(2)}^{\prime}$, $\mathscr{P}_{2,(2)}$ and magnetic resistor $R_{m,(2)}$ of another core with other cross section area $A_{2}$ and equivalent magnetic path length $l_{2}$ can be scaled from $\mathscr{P}_{1,(1)}^{\prime}, \mathscr{P}_{2,(1)}$ and $R_{m,(1)}$, given as:

$$
\begin{aligned}
& \mathscr{P}_{1,(2)}^{\prime}=\mathscr{P}_{1,(1)}^{\prime} \cdot \frac{A_{2}}{A_{1}} \cdot \frac{l_{1}}{l_{2}} \\
& \mathscr{P}_{2,(2)}=\mathscr{P}_{2,(1)} \cdot \frac{A_{2}}{A_{1}} \cdot \frac{l_{1}}{l_{2}} \\
& R_{m,(2)}=R_{m,(2)} \cdot \frac{A_{1}}{A_{2}} \cdot \frac{l_{2}}{l_{1}}
\end{aligned}
$$

The verification is firstly carried out on the toroidal core with shape code "R 25.3 x 14.8 x 10.0", the geometry difference compared to the previous core sample " $\mathrm{R} 20.0$ $\mathrm{x} 10.0 \times 7.00$ " is visible in Fig. 22a. PWM excitation of $50 \mathrm{kHz}$ switching frequency and different zero-voltage period is applied, while the DC voltage sources are configured to obtain $\hat{H}=20 \mathrm{~A} / \mathrm{m}$ peak equivalent field strength, which is similar to the case presented in the section IV-A. The comparison between simulated and measured core loss power are displayed in Fig. 22b, where only small error exists. Please note that the scaling can be also applied to the model of a complex core, where separate parts of the core are represented by a circuit of multiple permeances.

The E-core with shape code "E 20/10/6" (product number "B66311G0000X187") is taken as the next verification case as shown in Fig. 23a. The circuit model is established according to the real geometry, as shown in Fig. 23b. Each of the 


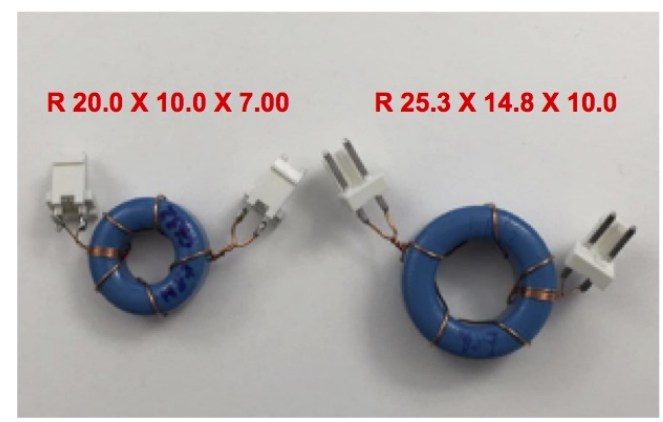

(a)

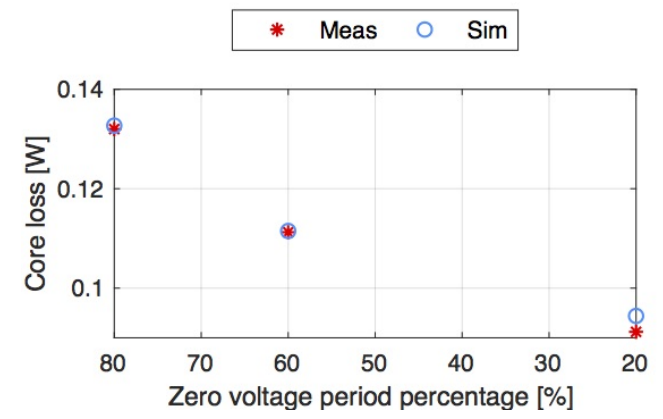

(b)

Fig. 22: (a) Core sample "R 25.3 x 14.8 x 10.0" and "R 20.0 x 10.0 x 7.00" of different geometries; (b) Core loss comparison between measurement and simulation under $50 \mathrm{kHz}$ PWM excitation with peak field strength $\hat{H}=20 A / m$ and different percentage of zero-voltage period $T_{0}$, obtained from the core "R 25.3 x 14.8 x 10.0".

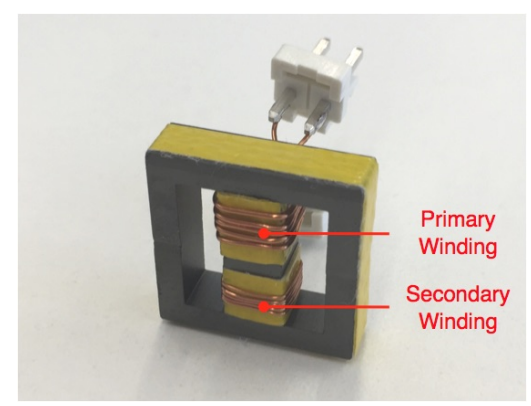

(a)

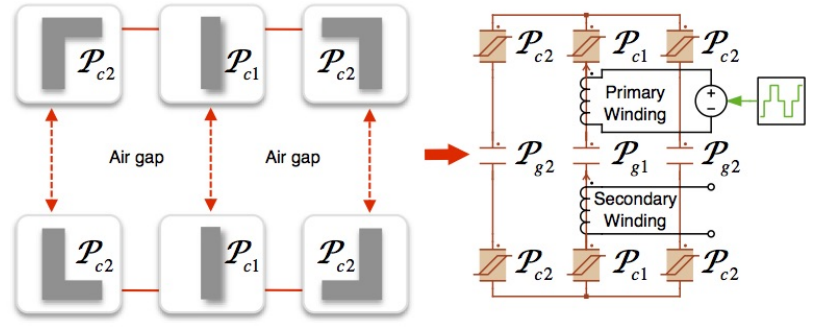

(b)

Fig. 23: (a) E-shape core sample "E 20/10/6"; (b) Simulation model with separate permeances representing middle-, side limbs and air gap of the core.

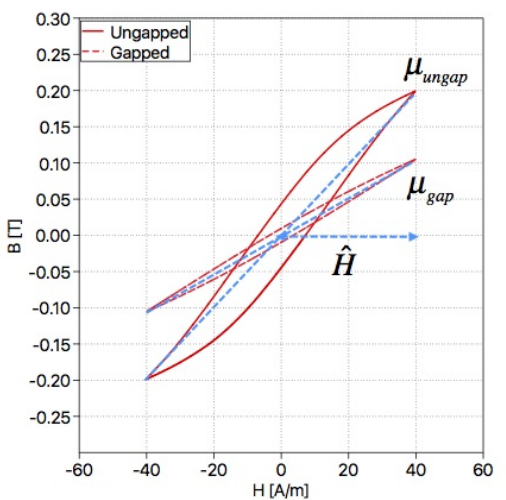

Fig. 24: $B-H$ characteristic measured from toroidal core without air gap (peak flux density $\hat{B}_{\text {ungap }}$ ) and E-core with tiny air gap (peak flux density $\hat{B}_{\text {gap }}$ ), under $500 \mathrm{~Hz}$ sinusoidal excitation.

permeances $\mathscr{P}_{\mathrm{c} 1}$ and $\mathscr{P}_{\mathrm{c} 2}$ represents one single part of the core, which has internal structure shown in Fig. 19. The equivalent geometrical parameters (cross section area and magnetic path length) are calculated following the formula proposed by [24]. All the values $\mathscr{P}_{1}^{\prime}, \mathscr{P}_{2}$ and $R_{m}$ for the individual permeances are scaled by the geometry of the corresponding part of the E-core from that obtained previously using the toroidal core sample, following equations (14) (16). Please note that there is no new experimental measurement needed for parametrization of the core pemeances $\mathscr{P}_{\mathrm{c} 1}$ and $\mathscr{P}_{\mathrm{c} 2}$, where the core material characteristic is included.

Considering the accuracy limitation of the test setup in measuring gapped cores, where the phase shift $\Phi_{\mathrm{v}-\mathrm{i}}$ becomes close to $90^{\circ}$ and result in extremely high $\tan \left(\Phi_{\mathrm{v}-\mathrm{i}}\right)$ in equation (1), the two E-core parts are tightly pressed together. Nevertheless, tiny air gap still exist in between, depending on the treatment of the joining surface during manufacture process. The length $l_{\mathrm{g}}$ of the tiny air gap between the two Ecore parts is difficult to measure, but can be estimated using the measured frequency independent $B-H$ characteristic of the Ecore and that of the toroidal core: The air gap result in different shape of the static hysteresis loop obtained under $500 \mathrm{~Hz}$ sinusoidal excitation, in comparison to that of the toroidal core, as demonstrated in Fig. 24. With the same equivalent peak field strength $\hat{H}=40 \mathrm{~A} / \mathrm{m}$, the loop of toroidal core and Ecore exhibits different equivalent permeability $\mu_{\text {ungap }}$ and $\mu_{\text {gap }}$ (slope of the virtual straight line connecting the two peaks), respectively. $l_{\mathrm{g}}$ can be calculated as the equations in below. Please note that the air gap has linear $B-H$ characteristic, so that the permeance obtained in the two equation $s$ above remains constant for different operating conditions.

$$
l_{\mathrm{g}}=\frac{1}{2} \cdot \mu_{0} \cdot l_{e} \cdot\left(\frac{1}{\mu_{\text {gap }}}-\frac{1}{\mu_{\text {ungap }}}\right)=4.5 \mu \mathrm{m}
$$

where $l_{\mathrm{e}}$ is the equivalent magnetic path length of the Ecore provided by the datasheet. In the circuit model shown in Fig. 23b, the air gap on the middle limb is represented by the constant permeance $\mathscr{P}_{\mathrm{g} 1}$ and that on the side limbs by $\mathscr{P}_{\mathrm{g} 2}$. Please note that $\mathscr{P}_{\mathrm{g} 1}$ and $\mathscr{P}_{\mathrm{g} 2}$ has the same length $l_{g}$, while 

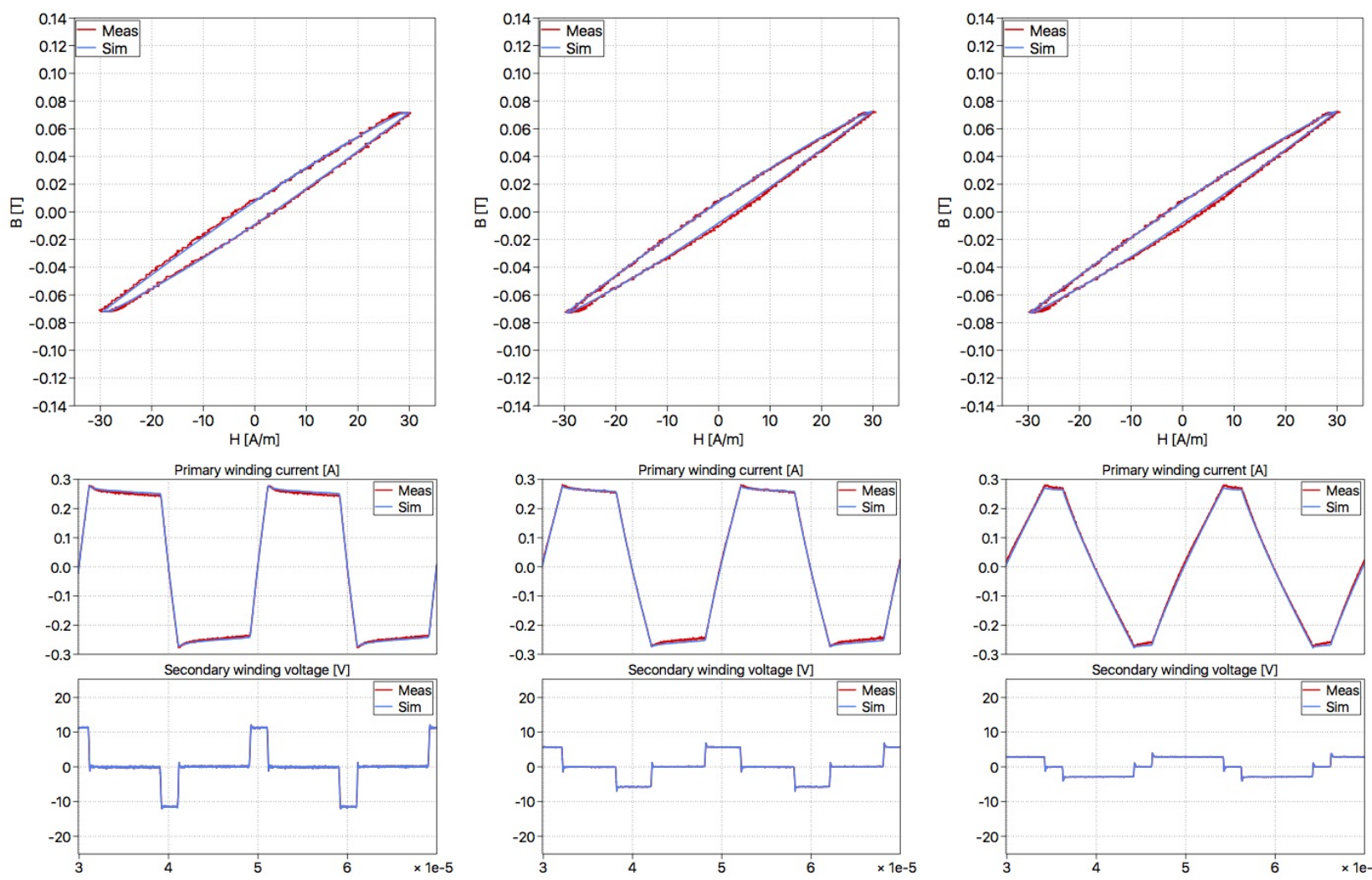

(a)

(b)

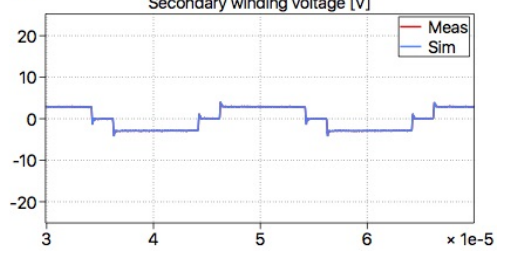

(c)

Fig. 25: $B-H$ characteristic and time domain waveform comparison of E-core "E 20/10/6" between measurement and simulation under $50 \mathrm{kHz}$ PWM excitation with equivalent peak field strength $\hat{H}=30 \mathrm{~A} / \mathrm{m}$ and different percentage of zerovoltage period $T_{0}$ (a) $80 \%$; (b) $60 \%$; (c) $20 \%$.

the cross section area are configured to be the same as $\mathscr{P}_{\mathrm{c} 1}$ and $\mathscr{P}_{\mathrm{c} 2}$, respectively.

$$
\mathscr{P}_{\mathrm{g} 1}=\mu_{0} \cdot \frac{A_{c 1}}{l_{g}}, \mathscr{P}_{\mathrm{g} 2}=\mu_{0} \cdot \frac{A_{c 2}}{l_{g}}
$$

The verification is still carried out under PWM excitation with different length of zero-voltage period, the DC voltage output of $V_{3}$ and $V_{4}$ are configured to generated unbiased $B-$ $H$ loop with peak field strength $\hat{H}=30 \mathrm{~A} / \mathrm{m}$. The time domain waveforms and the $B-H$ characteristic are compared between measurement and simulation in Fig. 25. Due to the presence of the tiny air gap, the $B-H$ characteristic has lower loop area and slope, in comparison to that of the toroidal core in Fig. 18. The influence of the zero-voltage period on the core loss is well reflected by the proposed model with maximum error of $13.5 \%$, as demonstrated in Fig. 26. The error is larger than the case of the toroidal core presented in Fig. 17, which can be ascribed to the estimation error of the air gap length $l_{g}$.

\section{F. Verification on core sample of different ferrite material}

In the last group of verification schemes, the model is verified with another ferrite material N30 from EPCOS. The toroidal core with shape code " $\mathrm{R} 16.0 \times \mathrm{X} 9.60 \times$ 6.30" is

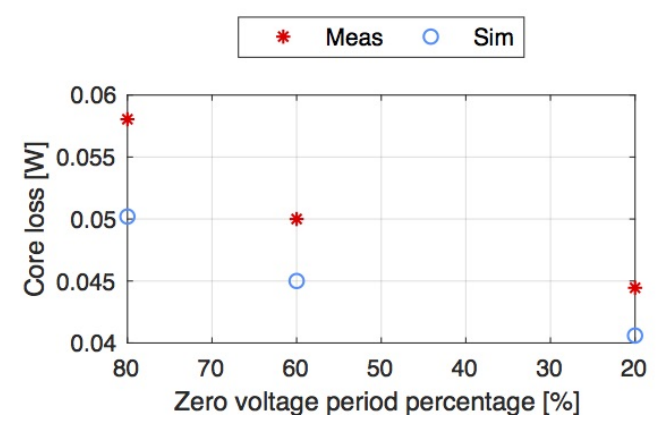

Fig. 26: Core loss comparison of E-core "E 20/10/6" between measurement and simulation under $50 \mathrm{kHz}$ PWM excitation with equivalent peak field strength $\hat{H}=30 \mathrm{~A} / \mathrm{m}$ and different percentage of zero-voltage period $T_{0}$.

taken as sample. Again $50 \mathrm{kHz}$ PWM excitation with different zero-voltage period is applied to the core sample, in which case the relaxation effect can be obviously observed. The model is parametrised using the measurement with $80 \%$ zero-voltage period and peak equivalent peak field strength $\hat{H}=20 A / m$. The resulted parameters are $\mathscr{P}_{2}=3.79 \cdot 10^{-7} \mathrm{H}$ and $R_{m}=2.43 \Omega^{-1}$, which are constant since measurement of one field strength amplitude is taken for parametrization. The 

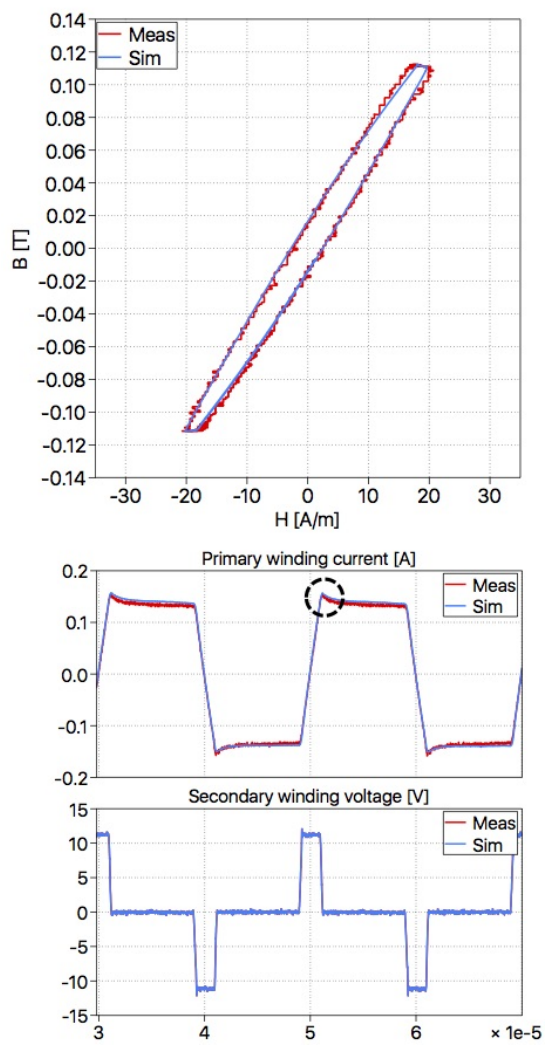

(a)
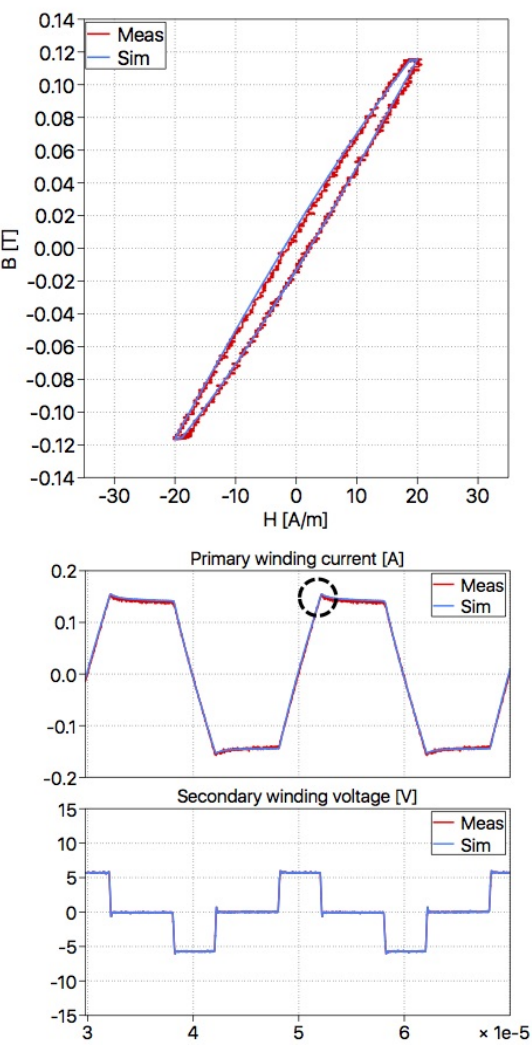

(b)
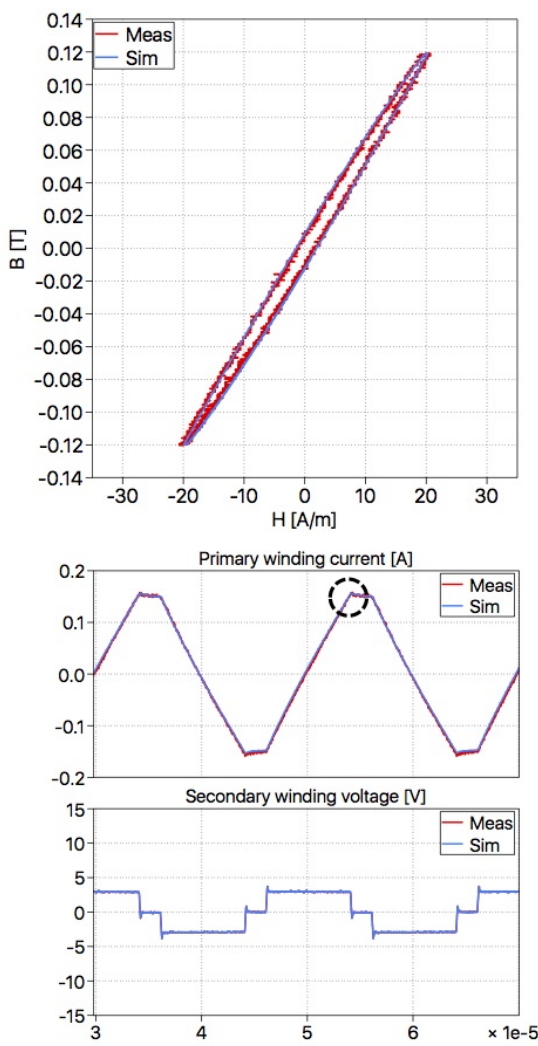

(c)

Fig. 27: $B-H$ characteristic and time domain waveform comparison between measurement and simulation of ferrite material N30 under $50 \mathrm{kHz}$ PWM excitation with peak field strength $\hat{H}=20 \mathrm{~A} / \mathrm{m}$ and different percentage of zero-voltage period $T_{0}$ (a) $80 \%$; (b) $60 \%$; (c) $20 \%$.

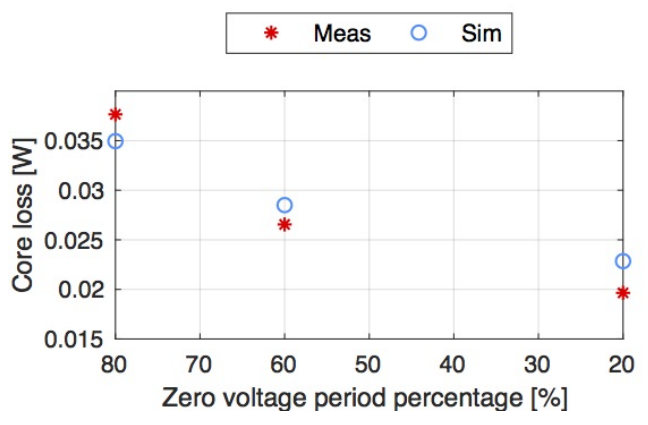

Fig. 28: Core loss comparison between measurement and simulation of ferrite material $\mathrm{N} 30$ under $50 \mathrm{kHz}$ PWM excitation with peak field strength $\hat{H}=20 \mathrm{~A} / \mathrm{m}$ and different percentage of zero-voltage period $T_{0}$.

comparison between simulation and measurement regarding the $B-H$ characteristic, the time domain waveform and the core loss power are presented in Fig. 27 and Fig. 28, respectively. In comparison to the ferrite material N87, the $B-H$ loop of N30 exhibits significantly different shape, and still, good match has been achieved by the proposed model.

This work focus on frequency dependent core loss of ferrite materials, whose electrical conductivity is extremely low. In this way, the conductivity related eddy current effect can be neglected in the frequency range that has been considered ( $\leq$ $50 \mathrm{kHz}$ ), and the relaxation effect which is not related to the material conductivity can be clearly analyzed. For the other metal based materials (e.g. metal powders or nanocrystalline), the frequency dependent core loss can be dominated by the eddy current effect, which is however, not in scope of this work.

\section{APPLICATION OF THE PROPOSED MODEL}

One application of the proposed model is the optimization of the magnetic component, which can be carried out in an intuitive way, taking into account the its desired operating condition in the target power converter system. Since optimization method is not in scope of this work, only a simplified workflow is demonstrated in Fig. 29 and described in below:

- (1) At first, the required material characteristic under low frequency sinusoidal- and high frequency PWM excitation is obtained using a toroidal core sample.

- (2) Secondly the permeance model is parametrized following the procedure introduced in section III.

- (3) Then, the iterative optimization of the magnetic component can be started. In each iteration, a different core geometry and turns number of the windings are chosen. 


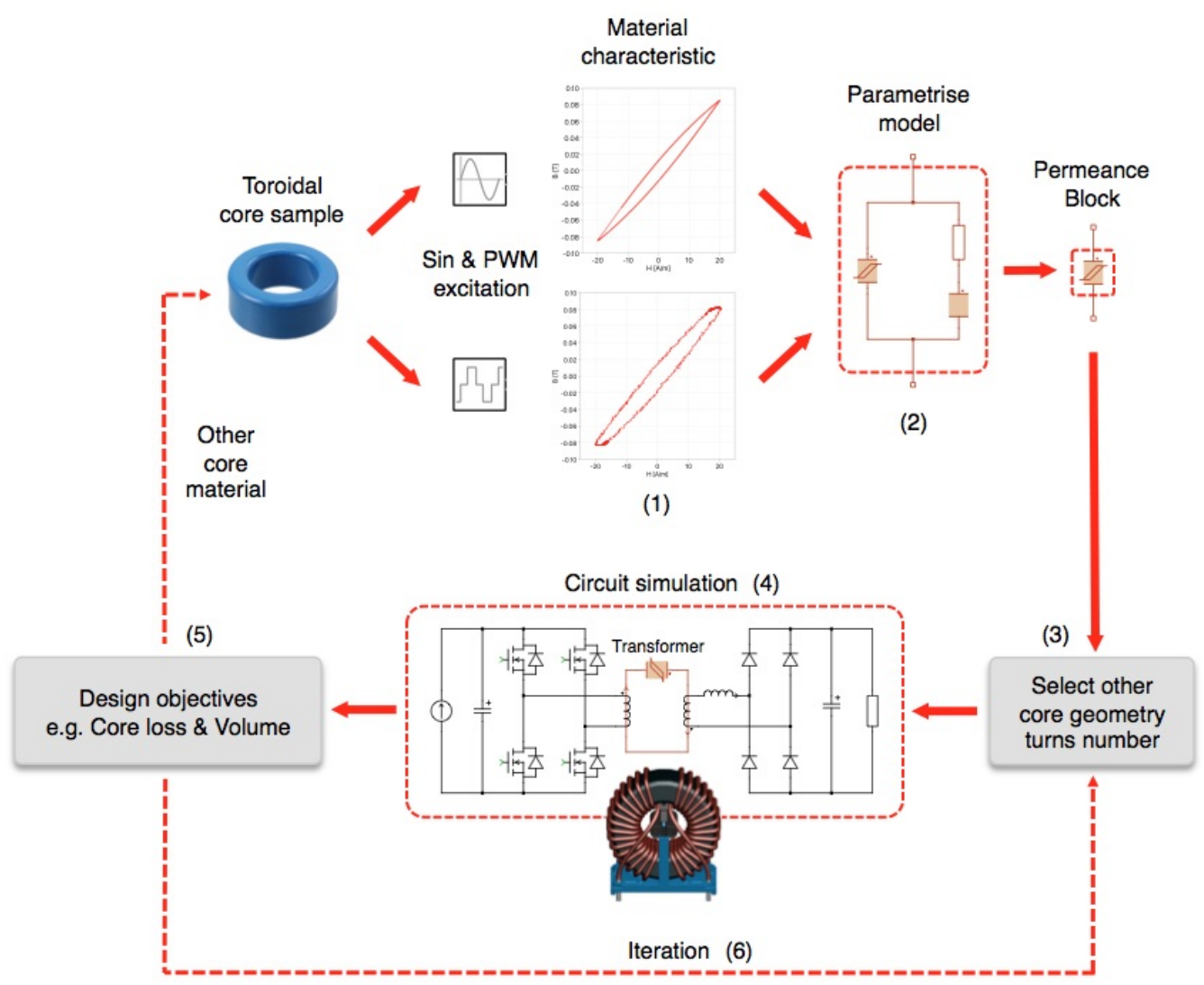

Fig. 29: Work flow using the proposed model to optimize magnetic component design

- (4) The permeance block is integrated into the circuit simulation environment of the target power converter system and scaled by the core geometry, according to the description in section IV-E. Also the winding components is configured by the desired turns number. The circuit model is simulated in time domain and the core loss power is obtained.

- (5) Taking the simulated core loss power and the volume of the selected core in the current iteration, certain objective function as trade-off between power loss and volume is evaluated.

- (6) The output of the objective function indicates the direction to change the core geometry and winding turns number, afterwards the new core geometry and winding turns number are applied to the circuit model in the next iteration.

\section{CONCLUSION}

This work proposes a modeling approach for frequencydependent core loss of ferrite core materials, which takes into account both frequency-independent magnetic hysteresis and frequency-dependent relaxation effect. The model can be seamlessly integrated into system-level circuit simulations, and can be easily extended to different geometries. The fidelity of the proposed model has been verified under PWM excitations of different switching frequencies, zero voltage-period and duty-cycle. With the proposed model, the power electronic designers are able to get intuitive insight to the magnetic core loss of magnetic components using ferrite materials, in relation to the individual operating conditions. Based on the simulation result, improvement or optimization on the magnetic component itself or the power electronic circuitry can be carried out in an efficiently way.

\section{ACKNOWLEDGMENT}

This project has been supported in the frame of the ECPE Joint Research Programme.

\section{REFERENCES}

[1] J. B. Goodenough, "Summary of losses in magnetic materials," in IEEE Transactions on Magnetics, vol. 38, no. 5, 2002, pp. 3398-3408.

[2] J. Reinert, A. Brockmeyer, and R. W. A. A. D. Doncker, "Calculation of losses in ferro- and ferrimagnetic materials based on the modified steinmetz equation," in IEEE Transactions on Industry Applications, vol. 37, no. 4, 2001, pp. 1055-1061.

[3] C. R. Sullivan, J. H. Harris, and E. Herbert, "Core loss predictions for general pwm waveforms from a simplified set of measured data," in 25th Annual IEEE Applied Power Electronics Conference and Exposition (APEC), 2010, pp. 1048-1055.

[4] J. Muehlethaler, J. Biela, J. W. Kolar, and A. E. Ecklebe, "Improved core-loss calculation for magnetic components employed in power electronic systems," in IEEE Transactions on Power Electronics, vol. 27, no. 2, 2012, pp. 964-973.

[5] S. Barg, K. Ammous, H. Mejbri, and A. Ammous, "An improved empirical formulation for magnetic core losses estimation under nonsinusoidal induction," in IEEE Transactions on Power Electronics, vol. 32, no. 3, 2017, pp. 2146-2154.

[6] V. Basso, "Hysteresis and relaxation effects in magnetic materials," in IEEE Transactions on Magnetics, vol. 36, no. 5, 2000, pp. 3176-3181. 
[7] A. Abramovitz and S. Ben-Yaakov, "Rgse-based spice model of ferrite core losses," in IEEE Transactions on Power Electronics, vol. 33, no. 4, 2018, pp. 2825-2831.

[8] I. Mayergoyz, Mathematical Models of Hysteresis and Their Applications. Academic Press, 2003.

[9] J. T. Hsu and K. D. T. Ngo, "A hammerstein-based dynamic model for hysteresis phenomenon," in IEEE Transactions on Power Electronics, vol. 12 , no. 3, 1997, pp. 406-413.

[10] M. Luo, D. Dujic, and J. Allmeling, "Modeling frequency independent hysteresis effects of ferrite core materials using permeance-capacitance analogy for system-level circuit simulations," in IEEE Transactions on Power Electronics, 2018, Early Access.

[11] J. G. Zhu, S. Y. R. Hui, and V. S. Ramsden, "A dynamic equivalent circuit model for solid magnetic cores for high switching frequency operations," in IEEE Transactions on Power Electronics, vol. 10, no. 6 , 1995, pp. 791-795.

[12] J. van Vlerken and P. G. Blanken, "Lumped modelling of rotary transformers, heads and electronics for helical-scan recording," in IEEE Transactions on Magnetics, vol. 31, no. 2, 1995, pp. 1050-1055.

[13] L. Dalessandro, W. G. H. Odendaal, and J. W. Kolar, "Hf characterization and nonlinear modeling of a gapped toroidal magnetic structure," in IEEE Transactions on Power Electronics, vol. 21, no. 5, 2006, pp 1167-1175.

[14] D. Hamill, "Lumped equivalent circuits of magnetic components: the gyrator-capacitor approach," in IEEE Transactions on Power Electronics, vol. 8, 1994, pp. 97-103.

[15] J. Allmeling, W. Hammer, and J. Schönberger, "Transient simulation of magnetic circuits using the permeance-capacitance analogy," in Contro and Modeling for Power Electronics (COMPEL), IEEE 13th Workshop on, 2012.

[16] D. Tan, J. L. Vollin, and S. M. Cuk, "A practical approach for magnetic core-loss characterization," in IEEE Transactions on Power Electronics, vol. 10, no. 2, 1995, pp. 124-130.

[17] Y. Han, G. Cheung, A. Li, C. R. Sullivan, and D. J. Perreault, "Evaluation of magnetic materials for very hight frequency power applications," in IEEE Transactions on Power Electronics, 425-435, Ed., vol. 27, no. 1, 2012.

[18] M. Mu, Q. Li, D. J. Gilham, F. C. Lee, and K. D. T. Ngo, "New core los measurement method for high-frequency magnetic materials," in IEEE Transactions on Power Electronics, vol. 29, no. 8, 2014, pp. 4374-4381.

[19] D. Hou, M. Mu, F. C. Lee, and Q. Li, "New high-frequency core loss measurement method with practial cancellation concept," in IEEE Transactions on Power Electronics, vol. 32, no. 4, 2017, pp. 2987-2994.

[20] J. Muehlethaler, J. Biela, and J. W. Kolar, "Core losses under dc bias condition based on steinmetz parameters," in IEEE Transactions on Power Electronics, vol. 27, no. 2, 2012, pp. 953-963.

[21] TDK, "Epcos data book 2013: Ferrites and accesories," 2013.

[22] [Online]. Available: http://www.vishay.com/landingpage/extreme/2014/shunt

[23] M. Luo, D. Dujic, and J. Allmeling, "Permeance based modeling of magnetic hysteresis with inclusion of eddy current effect," in 2018 IEEE Applied Power Electronics Conference and Exposition (APEC), 2018.

[24] C. Marxgut, J. Muehlethaler, F. Krismer, and J. W. Kolar, "Multiobjective optimization of ultraflat magnetic components with pcb-integrated core," in IEEE Transactions on Power Electronics, vol. 28, no. 7, 2013 , pp. 3591-3602.

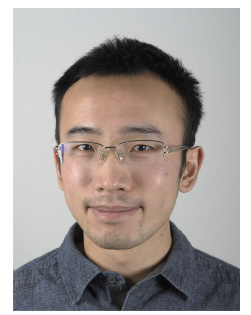

Min Luo Min Luo (M'13) was born in Beijing, China, in 1986. He received the B.S. degree in electrical engineering from Tsinghua University, Beijing, China, in 2009 and M.S. degree in electrical power engineering from RWTH Aachen University, Aachen, Germany, in 2012. Since 2012 he has been with Plexim as application engineer, working on the software PLECS for fast simulation of powe electronic systems. In 2014 he joined Power Electronics Laboratory of École Polytechnique Fédérale de Lausanne (EPFL) in Lausanne, Switzerland as external doctoral research assistant, pursuing his $\mathrm{PhD}$ degree. His current research interest include dynamic modelling of magnetic components and real-time HIL simulation of power converters.

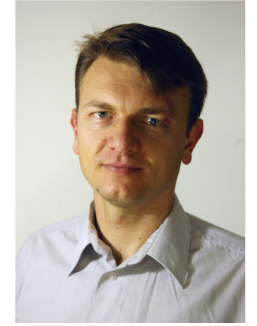

Drazen Dujic Drazen Dujic (S'03-M'09-SM'12) received the Dipl.-Ing. and M.Sc. degrees from the University of Novi Sad, Novi Sad, Serbia, in 2002 and 2005, respectively, and the Ph.D. degree from the Liverpool John Moores University, Liverpool, U.K., in 2008, all in electrical engineering. From 2002 to 2006, he was with the Department of Electrical Engineering, University of Novi Sad as a Research Assistant, and from 2006 to 2009 with Liverpool John Moores University as a Research Associate. From 2009 till 2013, he was with ABB Corporate Research Centre, Switzerland, as a Principal Scientist working on the power electronics projects spanning the range from low-voltage/power SMPS in below kilowatt range to medium voltage high-power converters in a megawatt range. During 2010-2011, he was a member of a project team responsible for the development of the worlds first power electronic traction transformer (PETT) successfully commissioned on the locomotive. From 2013 till 2014, he was with ABB Medium Voltage Drives, Turgi, Switzerland, as R\&D Platform Manager, responsible for ABB's largest IGCT based medium voltage drive - ACS6000. He is currently with Ecole Polytechnique Federale de Lausanne EPFL, Lausanne, Switzerland, as an Assistant Professor and the Director of the Power Electronics Laboratory. His current research interests include the areas of design and control of advanced high-power electronics systems and high performance drives. He has authored or coauthored more than 80 scientific publications and has filed eleven patents. He is an Associate Editor for IEEE Transactions on Industrial Electronics, IEEE Transaction on Power Electronics and IET Electric Power Applications. He has received the First Prize Paper Award by the Electric Machines Committee of the IEEE Industrial Electronics Society at IECON-2007. In 2014 he has received the Isao Takahashi Power Electronics Award for outstanding achievement in power electronics.

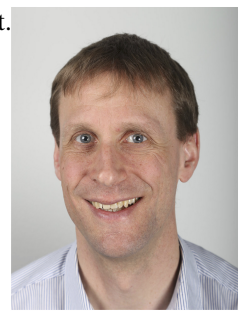

Jost Allmeling Jost Allmeling (S'98-M'03) was born in Hamburg, Germany, in 1972. He received the M.S. degree in electrical engineering from Aachen University, Aachen, Germany, in 1996 and the Ph.D. degree from the Swiss Federal Institute of Technology (ETH), Zurich, Switzerland, in 2001. In 1996, he became a Research Associate at the Power Electronics Laboratory at ETH. From 2001 until 2003, he was with the Power Systems Laboratory at ETH, as a Post-Doctoral Researcher. In 2002, Jost co-founded Plexim, a spin-off company from ETH Zurich that develops the software PLECS for fast simulation of powe electronic systems. Currently, he is the Managing Director of Plexim. His research interests include simulation of power electronics, modeling electrical and mechanical components, real-time HIL simulation and inverter control. 